\title{
Asymptotic Analysis of Acoustic Waves in a Porous Medium: Micro-Incompressible Flow
}

\author{
Jose Diaz-Alban * and Nader Masmoudi ${ }^{\dagger}$ \\ October 28, 2013
}

Key words: Acoustic waves, Compressible Navier-Stokes, Stokes, porous medium, Bloch decomposition, boundary layers.

AMS subject classification: 35B27, 35B40, 76N20, 76M50.

\begin{abstract}
This is the second in a series of three papers which studies acoustic waves governed by the linearized compressible Stokes equations in a porous medium. In particular, we want to analyze the simultaneous inviscid and high frequency limits of fluid flows in a porous medium. The presence of time-space boundary layers decouples the flow into an incompressible (that we call micro-incompressible) and an acoustic part (that we call micro-acoustic) on the microscopic scale. While this paper employs the two-scale methods used in our first paper [10], the present boundary layer phenomenon requires additional weak convergence tools. Using the Bloch decomposition, we introduce modified Helmholtz operators, enabling us to split the flow into its micro-incompressible and micro-acoustic parts. Closed equations for the micro-incompressible flow are obtained using two-scale convergence, while closed equations for the micro-acoustic flow are given in our forthcoming paper.
\end{abstract}

\section{Introduction}

The homogenization of the Stokes operator in a porous medium is well studied. We refer the interested reader to text books $[3,24,16]$ for some formal developments and to $[25,1,20]$ for some rigorous mathematical results. However, there are fewer works dealing with the homogenization of the acoustic system or of the compressible Navier-Stokes system (see for instance [7, 19, 11, 18, 29]). Here, we continue the study of the homogenization of the compressible Navier-Stokes system linearized around $(1,0)$ in a porous medium which was started in [10].

\footnotetext{
*josediaz@cims.nyu.edu,

${ }^{\dagger}$ masmoudi@cims.nyu.edu, Courant Institute of Mathematical Sciences. Partially supported by NSF grant DMS-1211806
} 
The linearized compressible Navier-Stokes equations in a porous medium read

$$
\left\{\begin{aligned}
\partial_{t} p^{\varepsilon}+\operatorname{div} u^{\varepsilon} & =0 \quad(0, \infty) \times \mathbb{R}_{\varepsilon}^{d} \\
\partial_{t} u^{\varepsilon}-\varepsilon^{\beta} \triangle u^{\varepsilon}+\nabla p^{\varepsilon} & =0 \\
u^{\varepsilon} & =0 \quad \partial \mathbb{R}_{\varepsilon}^{d} \\
p^{\varepsilon}(t=0) & =b^{\varepsilon} \\
u^{\varepsilon}(t=0) & =a^{\varepsilon}
\end{aligned}\right.
$$

where $p^{\varepsilon}$ and $u^{\varepsilon}$ are the pressure and velocity of the fluid, and $\varepsilon^{\beta}$ is the viscosity. Here, $\mathbb{R}_{\varepsilon}^{d}$ is the porous medium formed by periodic repetition of an elementary fluid cell which has been shrunk to size $\varepsilon$ (see section 2.1). Finally, $1<\beta$ measures the relative importance of the viscous effect to the size of each cell. We refer the reader to [10] for a complete study of the case of strongly viscous flow $(\beta \leq 1)$ and for a derivation of this model.

In the case of $1<\beta$, the presence of time-space boundary layers decouples the flow into an incompressible (that we call micro-incompressible, see Definition 3.5) and an acoustic part (that we call micro-acoustic, see Definition 3.5) on the microscopic scale. We remark that this is in contrast to the case of $\beta \leq 1$, where not only is the boundary layer in space absent, but the boundary layers in time decouples the velocity and pressure when $\varepsilon$ goes to zero (see [10]). In order to motivate the goals of this paper, we begin with a formal approach to the case when $\beta=2$ (we refer the interested reader to [24] and [19] for a formal and rigorous study when $\beta=2)$. Formal asymptotic expansions expressing $\left(p^{\varepsilon}, u^{\varepsilon}\right)$ in the variables $(t, x, y), y=\frac{x}{\varepsilon}$, yields the limiting terms $\left(p_{0}, u_{0}\right)$ and the associated two-scale system

$$
\left\{\begin{array}{rlrl}
\left|Y_{f}\right| \partial_{t} p_{0}+\operatorname{div} x \int_{Y_{f}} u_{0} & =0 & & \text { in } \mathbb{R}^{d} \\
\partial_{t} u_{0}-\triangle_{y} u+\nabla_{x} p_{0}+\nabla_{y} p_{1} & =0 & & \\
\operatorname{div}_{y} u_{0} & =0 & & \\
\nabla_{y} p_{0} & =0 & & \mathbb{R}^{d} \times Y_{f} \\
u_{0} & =0 & & \\
\int_{Y_{f}} p_{1} & =0 & & \\
p_{0}(t=0) & =b(x) & \\
u_{0}(t=0) & =P_{y} a_{0}(x, y) & \\
y \rightarrow\left(p_{1}, u_{0}\right) & \text { is } Y \text {-periodic } &
\end{array}\right.
$$

Here $p_{1}$ is the Lagrange multiplier corresponding to the incompressibility of $u_{0}$ and $P_{y}$ is the divergence free Helmholtz operator on the domain $Y_{f}$ (see Remark 3.1).

We notice that two-scale convergence captures the micro-incompressible part of the flow. What is not so apparent is the formation of a thin boundary layer in time of size $\varepsilon^{1 / 2}$ which traps the energy carried by the micro-acoustic part of the flow when $\varepsilon$ goes to zero. Throughout this paper we identify the micro-acoustic part of the flow as $\left(q^{\varepsilon}, v^{\varepsilon}\right)$. The overall behavior of the flow near initial time can be seen in the following diagram. 


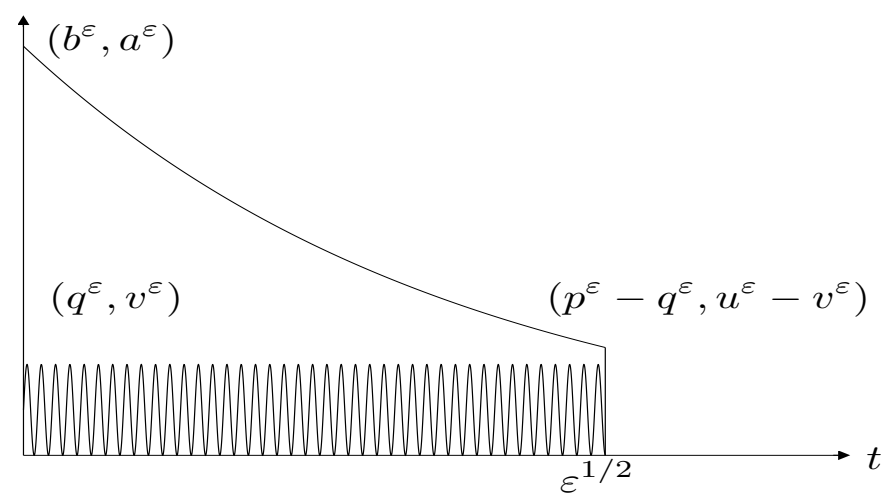

Figure 1: Behavior of the flow near $t=0$ when $\beta=2$.

We remark that the time behavior associated the micro-acoustic part of the flow is non-trivial. In fact, the initial layer in time of size $\varepsilon^{1 / 2}$ and time oscillations associated to $\left(q^{\varepsilon}, v^{\varepsilon}\right)$ are generated by the presence of a boundary layer in space of size $\varepsilon^{3 / 2}$.

This paper is devoted to the complete study of the micro-incompressible flow for $1<\beta$. Our goal is to describe the energy carried by the micro-incompressible part of the flow as $\varepsilon$ goes to zero. Mathematically, we wish to understand the local and total energies

$$
\begin{aligned}
e^{\varepsilon}(t, x) & =\left|p^{\varepsilon}(t, x)-q^{\varepsilon}(t, x)\right|^{2}+\left|u^{\varepsilon}(t, x)-v^{\varepsilon}(t, x)\right|^{2} \\
E^{\varepsilon}(t) & =\left\|p^{\varepsilon}(t)-q^{\varepsilon}(t)\right\|_{L^{2}\left(\mathbb{R}_{\varepsilon}^{d}\right)}^{2}+\left\|u^{\varepsilon}(t)-v^{\varepsilon}(t)\right\|_{L^{2}\left(\mathbb{R}_{\varepsilon}^{d}\right)}^{2}
\end{aligned}
$$

when fluid flow occurs in a porous medium and the limit $\varepsilon$ going to zero is taken. We note that the energy carried by the micro-acoustic part of the flow is subject of our final paper [9].

A complete understanding of the underlying time-space boundary layer phenomena is necessary in order to propose appropriate asymptotic expansions describing the energy carried by micro-incompressible flow. In the case $1<\beta<2$, we show that the boundary layer in time of size $\varepsilon^{2-\beta}$ decouples the microincompressible part of the flow when $\varepsilon$ goes to zero. More precisely, the usual formal two-scale expansions in the variables $(t, x, y), y=\frac{x}{\varepsilon}$, will describe the pressure $p^{\varepsilon}-q^{\varepsilon}$, but will fail to describe the velocity $u^{\varepsilon}-v^{\varepsilon}$ for all times. In order to describe the velocity near initial time, we need to introduce the time scale $\tau=t / \varepsilon^{2-\beta}$ during the homogenization process. Through this time rescaling, we deduce that the velocity associated to micro-incompressible flow is governed by the Stokes system (16) near initial time. The overall behavior of the flow near initial time can be seen in the following diagram.

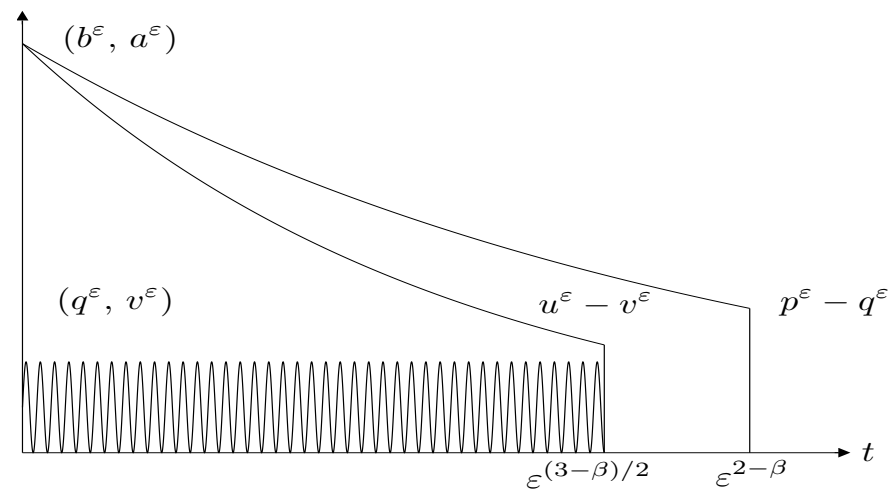


Figure 2: The behavior of the flow near $\mathrm{t}=0$ for $1<\beta<2$.

We make a few observations. First, we note that the boundary layer in time of size $\varepsilon^{2-\beta}$ remains from the strongly viscous regime (see [10]). Secondly, the time behavior that impacts the micro-acoustic part of the flow, namely the boundary layer in time of size $\varepsilon^{(3-\beta) / 2}$ and associated time oscillations are non-trivial and are generated by a boundary layer in space of size $\varepsilon^{(\beta+1) / 2}$. This time behavior associated to micro-acoustic flow is essentially given in [6] (see also [15] for a more precise construction) for the case $\beta=2$, and we refer the reader to [8], [9] for further details in the general case $1<\beta$. Lastly, we invite the reader to compare with Figure 1 and note that boundary layer in time of size $\varepsilon^{2-\beta}$ breaks when $\beta=2$, hence the coupling of the pressure and velocity of micro-incompressible flow.

When $2<\beta$, there is no boundary layer in time that impacts the micro-incompressible part of the flow. Nevertheless, using a formal approach to yield the asymptotic behavior of micro-incompressible flow yields the following information

$$
\lim _{\varepsilon \rightarrow 0+}\left(u^{\varepsilon}-v^{\varepsilon}\right)\left(t, x, \frac{x}{\varepsilon}\right)=u_{0}(\tau, x, y) \neq 0 \quad \text { on } \partial Y_{s}
$$

The failure of the no-slip condition on the microscopic level is due to the formation of a boundary layer in space of size $\varepsilon^{\beta / 2}$, which forms as $\varepsilon$ vanishes. It follows that linearized Prandtl boundary layer expansions are necessary for the case $2<\beta$, and we hope that the reader will find the presentation given here interesting in its own right. Using the Prandtl expansions, we show that this layer quickly dissipates the energy carried by the waves. That is, the energy carried by micro-incompressible flow is described by the closed equations obtained via the usual two-scale process.

In closing, we leave the reader with a few remarks concerning micro-incompressible flow. First, we note that while $\beta=2$ is in a sense a critical value, we do not assign definitions to the regimes $1<\beta<2$ and $2<\beta$. Also, while there is a boundary layer in space of size $\varepsilon^{(\beta+1) / 2}$ when $1<\beta$, we only briefly mentioned it since its impact is mainly related to micro-acoustic flow, which is the subject of our forthcoming paper [9]. Moreover, while this paper employs the two-scale methods used in our first paper, the present boundary layer phenomena requires additional weak convergence tools. For instance, as we mentioned earlier the Prandtl expansions are necessary for the case $2<\beta$, but also incorporated in the homogenization process is the Bloch decomposition. We refer the reader to [28], [5] and [12] as examples of just some of works involving the Bloch decomposition. Using the Bloch decomposition we introduce the modified Helmholtz operators $P_{M}^{\varepsilon}, Q_{M}^{\varepsilon}$ (see section 3.1), which in essence serve as the basis for the current article as well as our forthcoming work [9]. Indeed, it is through these modified operators that we are able to partition the flow into its incompressible and acoustic parts on the microscopic level. What's more, we believe the modified Helmholtz operators to be new and interesting in their own right.

\section{Preliminaries}

\subsection{The Domain}

Define $Y=(0,1)^{d}$ to be the unit open cube in $\mathbb{R}^{d}$, and let $Y_{s}$ be a closed smooth subset of $Y$ with strictly positive measure. By smoothness of $\partial Y_{s}$, we mean to take it as regular as needed. The domain $Y / Y_{s}$ is denoted by $Y_{f}$ and we refer to $Y_{s}, Y_{f}$ to be the solid and fluid parts of $Y$. Repeating $Y_{f}$ by $Y$-periodicity to all of $\mathbb{R}^{d}$ we get the fluid domain $\mathbb{R}_{0}$. The porous medium is now defined to be $\varepsilon \mathbb{R}_{0}$ which is denoted by $\mathbb{R}_{\varepsilon}^{d}$. We define $Y_{f}^{\varepsilon}, Y_{s}^{\varepsilon}$ to be $\varepsilon Y_{f}, \varepsilon Y_{s}$ and note

$$
\mathbb{R}_{\varepsilon}^{d}=\left\{x: x \in Y_{f}^{\varepsilon}+p, \quad \text { for some } p \in \mathbb{Z}^{d}\right\} .
$$




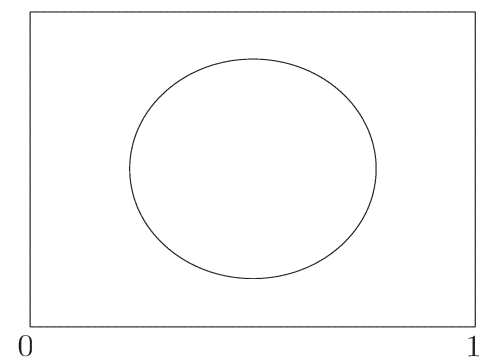

Figure 3: The elementary fluid cell

\subsection{Notations}

We define $B=[0,2 \pi)^{d}$ and we say that a function $f(x)$ defined on $\mathbb{R}_{0}^{d}$ is $\theta$-quasi periodic, for $\theta \in B$, provided

$$
f(x+p)=e^{i \theta \cdot p} f(x), \quad \forall p \in \mathbb{Z}^{d} .
$$

A function which is 0-quasi periodic, is simply referred to as $Y$-periodic.

The space of smooth functions which vanish at infinity is denoted by $D\left(\mathbb{R}^{d}\right)$, and for $\theta \in B, C_{\theta}^{k}\left(Y_{f}\right)$ is the space of differentiable functions up to order $k$ which are $\theta$-quasi periodic. We define the space $L_{\theta}^{2}\left(Y_{f}\right)$ to be all functions in $L_{l o c}^{2}\left(\mathbb{R}_{0}^{d}\right)$ satisfying (4). The Sobolev space $H_{\theta}^{k}\left(Y_{f}\right)$ is the space of the functions with derivatives up to order $k$ belonging to $L_{\theta}^{2}\left(Y_{f}\right)$ and the space $H_{0 \theta}^{k}\left(Y_{f}\right.$ is the subspace of functions in $H_{\theta}^{k}\left(Y_{f}\right)$ that vanish on the boundary $\partial Y_{s}^{\varepsilon}$. Also recall that the space $H_{\theta}^{-1}\left(Y_{f}\right)$ is defined by the norm

$$
\|f\|_{H_{\theta}^{-1}\left(Y_{f}\right)}=\sup _{\|u\|_{H_{0 \theta}^{1}\left(Y_{f}\right)}=1}|<f, u>|
$$

When $\theta=0$, we use the notations $C_{\#}^{k}\left(Y_{f}\right), L_{\#}^{2}\left(Y_{f}\right), H_{\#}^{k}\left(Y_{f}\right), H_{0 \#}^{k}\left(Y_{f}\right), H_{\#}^{-1}\left(Y_{f}\right)$. We denote $X^{d}$ to be the Banach space of vector valued functions of $d$-components, each component belonging to Banach space $X$. We note that all the Hilbert spaces mentioned in this paper are equipped with an inner product whose second component is conjugated, here $z^{*}$ denotes the complex conjugate of $z$.

Lastly, the we define the operators $[\cdot]_{1},[\cdot]_{2}$ acting on $\mathbb{C}^{d+1}$ as

$$
[z]_{1}=z_{1}, \quad[z]_{2}=z_{2},
$$

where $z=\left(z_{1}, z_{2}\right) \in \mathbb{C} \times \mathbb{C}^{d}$.

\subsection{Two-Scale Convergence}

Two-scale convergence is a notion first introduced by G. Nguetseng, [21], and later extended by G. Allaire [2], used to capture the parts of $f^{\varepsilon}$ which oscillate at frequency $\varepsilon^{-1}$. More precisely, two-scale convergence is a rigorous justification of the first term in formal two-scale expansions. In our problem two-scale convergence plays a significant role in understanding the local and total energies (3).

Definition 2.1 Let $f^{\varepsilon}(x), f_{0}(x, y)$ belong to $L^{2}\left(\mathbb{R}_{\varepsilon}^{d}\right), L^{2}\left(\mathbb{R}^{d} \times Y\right)$ respectively. We say $f^{\varepsilon}(x)$ two-scale converges to $f_{0}(x, y)$ (denoted $f^{\varepsilon} \stackrel{2-s}{\longrightarrow} f_{0}$ ) provided

$$
\int_{\mathbb{R}_{\varepsilon}^{d}} f^{\varepsilon}(x) \sigma\left(x, \frac{x}{\varepsilon}\right) \stackrel{\varepsilon \rightarrow 0}{\rightarrow} \int_{\mathbb{R}^{d}} \int_{Y_{f}} f_{0}(x, y) \sigma(x, y),
$$

for all $\sigma \in D\left(\mathbb{R}^{d} ; C_{\#}^{\infty}(Y)\right)$. 
Notice that if $f^{\varepsilon}(x)$ two-scale converges to $f_{0}(x, y)$ then the following always holds

$$
\lim _{\varepsilon \rightarrow 0}\left\|f^{\varepsilon}\right\|_{L^{2}\left(\mathbb{R}_{\varepsilon}^{d}\right)}^{2} \geq\left\|f_{0}\right\|_{L^{2}\left(\mathbb{R}^{d} \times Y_{f}\right)}^{2} .
$$

If equality holds, we have

Definition 2.2 We say $f^{\varepsilon}$ strongly two-scale converges to $f_{0}(x, y)$, denoted $f^{\varepsilon} \stackrel{2-s}{\rightarrow} f_{0}(x, y)$ provided $f^{\varepsilon}$ two-scale converges to $f_{0}$ and

$$
\lim _{\varepsilon \rightarrow 0}\left\|f^{\varepsilon}\right\|_{L^{2}\left(\mathbb{R}_{\varepsilon}^{d}\right)}^{2}=\left\|f_{0}\right\|_{L^{2}\left(\mathbb{R}^{d} \times Y\right)}^{2} .
$$

Strong two-scale convergence is interpreted as saying that all of the oscillations of $f^{\varepsilon}$ are of frequency $\varepsilon^{-1}$. This is due to the fact that strong two-scale convergence implies

$$
\lim _{\varepsilon \rightarrow 0}\left\|f^{\varepsilon}\right\|_{L^{2}\left(\mathbb{R}_{\varepsilon}^{d}\right)}=\lim _{\varepsilon \rightarrow 0}\left\|f_{0}\left(x, \frac{x}{\varepsilon}\right)\right\|_{L^{2}\left(\mathbb{R}_{\varepsilon}^{d}\right)}
$$

whenever $f_{0}$ is continuous in either the $x$ or $y$ variable. More generally, (5) holds provided $f_{0}$ is an admissible test function in the sense of Allaire (see definition 1.4 of [2]). We note that given $f_{0}(x, y)$, having continuity in at least one of its variables enables us to make sense of the quantity $f_{0}\left(x, \frac{x}{\varepsilon}\right)$. We refer the reader to Section 5 of [2] for further discussion and to [10] for a characterization of strong two-scale convergence.

If $f_{0}(x, y)$ is an admissible test function, then we can improve the convergence of (5). Indeed, we have the following

$$
\lim _{\varepsilon \rightarrow 0} \int_{\mathbb{R}_{\varepsilon}^{d}}\left|f^{\varepsilon}(x)-f_{0}\left(x, \frac{x}{\varepsilon}\right)\right|^{2}=0 .
$$

Finally, we can add a time dependence in all the limits we mentioned above. In particular if $f_{0} \in$ $C\left([0, T] ; L_{\#}^{2}\left(Y_{f} ; C\left(\mathbb{R}^{d}\right) \cap L^{2}\left(\mathbb{R}^{d}\right)\right)\right)$, then we have

$$
\lim _{\varepsilon \rightarrow 0}\left\|f_{0}\left(t, x, \frac{x}{\varepsilon}\right)\right\|_{L^{2}\left((0, T) \times \mathbb{R}_{\varepsilon}^{d}\right)}=\left\|f_{0}\right\|_{L^{2}\left((0, T) \times \mathbb{R}^{d} \times Y_{f}\right)} .
$$

The main result of two-scale convergence is the existence of a two-scale limit for bounded sequences in $L^{2}\left(\mathbb{R}_{\varepsilon}^{d}\right)$. The main result is stated below; for a proof we refer the reader to [21], [2].

Theorem 2.3 Let $f^{\varepsilon}$ belong to a bounded set of $L^{2}\left(\mathbb{R}_{\varepsilon}^{d}\right)$. Then there exist a subsequence of $\varepsilon$ (still denoted by $\varepsilon$ ) and a $Y$-periodic function $f_{0}(x, y) \in L^{2}\left(\mathbb{R}^{d} \times Y\right)$, such that $f^{\varepsilon}(x)$ two-scale converges to $f_{0}(x, y)$.

\subsection{The Bloch Decomposition of $A_{0}$}

In order to completely state the main results of this paper, we introduce the Bloch decomposition of the wave operator

$$
A_{0}=\left(\begin{array}{cc}
0 & \text { div } \\
\nabla & 0
\end{array}\right)
$$

on the domain $Y_{f}^{\varepsilon}$. Here, $\varepsilon$ will be fixed and one can think as if $\varepsilon=1$. We keep it in the notation for later use. We begin by defining

$$
L_{\oplus, \varepsilon}^{2}=f_{B} L_{\theta}^{2}\left(Y_{f}^{\varepsilon}\right) d \theta
$$

to be the space of all measurable mappings

$$
u: B \rightarrow L_{\theta}^{2}\left(Y_{f}^{\varepsilon}\right)
$$

with finite norm

$$
\|u\|_{L_{\oplus, \varepsilon}^{2}}^{2}=f_{B} f_{Y_{f}^{\varepsilon}}|u(x, \theta)|^{2} d x d \theta<\infty .
$$

Due to the presence of the holes in our problem, one uses the test functions $\phi \in C_{c}^{\infty}\left(\mathbb{R}_{\varepsilon}^{d}\right)$ to deduce the following proposition. The proof is essentially given in [23]. 
Proposition 2.4 The mapping $I^{\varepsilon}: L^{2}\left(\mathbb{R}_{\varepsilon}^{d}\right) \rightarrow L_{\oplus, \varepsilon}^{2}$ defined as

$$
\left(I^{\varepsilon} u\right)(x, \theta)=u_{\#}(x, \theta)=\sum_{p \in \mathbb{Z}^{d}} u(x-\varepsilon p) e^{i \theta \cdot p}
$$

is unitary. The inverse is given by

$$
\left(\left(I^{\varepsilon}\right)^{-1} u_{\#}\right)(x)=f_{B} u_{\#}(x, \theta) d \theta
$$

If $u, v$ belong to $L^{2}\left(\mathbb{R}_{\varepsilon}^{d}\right)$, then Parseval's formula holds,

$$
(u, v)_{L^{2}\left(\mathbb{R}_{\varepsilon}^{d}\right)}=\left(u_{\#}, v_{\#}\right)_{L_{\oplus, \varepsilon}^{2}}
$$

We now study the spectral problem with Neumann boundary conditions. More precisely, we define for each $\theta \in B$, the non-decreasing eigenvalues $\left\{\mu_{k}^{2}(\theta)\right\}_{1 \leq k}$ and eigenvectors $\left\{\phi_{k}(x, \theta)\right\}_{1 \leq k}$ satisfying

$$
\left\{\begin{aligned}
-\triangle \phi_{k}(\theta) & =\mu_{k}^{2}(\theta) \phi_{k}(\theta) & & \text { in } Y_{f} \\
\frac{\partial \phi_{k}(\theta)}{\partial \nu} & =0 & & \text { on } \partial Y_{s} \\
\phi_{k}(x+p, \theta) & =e^{i \theta \cdot p} \phi_{k}(x, \theta), & &
\end{aligned}\right.
$$

The following proposition summarizes some of the basic properties of the eigenvalues and eigenfunctions $\mu_{k}(\theta), \phi_{k}(\theta)$. A proof is provided in subsection 4.1.

Proposition 2.5 For each $\theta \in[0,2 \pi)^{d}$ there exist $\mu_{k}(\theta), \phi_{k}(\theta), k=1,2,3 \ldots$ satisfying $(6)$ such that

$$
0 \leq \mu_{1}^{2}(\theta) \leq \mu_{2}^{2}(\theta) \leq \ldots \leq \mu_{k}^{2}(\theta) \ldots \stackrel{k \rightarrow \infty}{\rightarrow} \infty
$$

Moreover:

(i) $\mu_{k}(\theta)$ belongs to $C_{\#}(B)$ and satisfies

$$
\sup _{\substack{\theta, \xi \\ \theta \neq \xi}} \frac{\left|\mu_{k}(\theta)-\mu_{k}(\xi)\right|}{|\theta-\xi|} \leq 1
$$

(ii) $0=\inf _{\theta \in B} \mu_{1}(\theta)<\inf _{\theta \in B} \mu_{2}(\theta) \leq \ldots \inf _{\theta \in B} \mu_{k}(\theta) \leq \ldots \stackrel{k \rightarrow \infty}{\rightarrow}$. Moreover, $\mu_{1}(0)=0$.

(iii) The principle eigenvalue $\mu_{1}(\theta)$ is simple in a neighborhood of the origin.

(iv) For all $\theta \in B$, the eigenvectors $\left\{\phi_{k}(\theta)\right\}_{1 \leq k}$ form an orthonormal basis of $L_{\theta}^{2}\left(Y_{f}\right)$. For $\theta \neq 0$, the sequence $\left\{\frac{\nabla \phi_{k}(\theta)}{\mu_{k}(\theta)}\right\}_{1 \leq k}$ forms an orthonormal basis of $\mathcal{H}_{\theta}\left(Y_{f}\right)$ where

$$
\mathcal{H}_{\theta}\left(Y_{f}\right)=\left\{u \in L_{\theta}^{2}\left(Y_{f}\right)^{d}: u=\nabla p \text { for some } p \in H_{\theta}^{1}\left(Y_{f}\right)\right\}
$$


(v) When $\theta=0$, the sequences $\left\{\phi_{k}(0)\right\}_{1<k},\left\{\frac{\nabla \phi_{k}(0)}{\mu_{k}(0)}\right\}_{1<k}$ form an orthonormal basis of $L_{\#}^{2}\left(Y_{f}\right) / \mathbb{R}$ and $\mathcal{H}_{\#}\left(Y_{f}\right)$ respectively where

$$
\begin{aligned}
L_{\#}^{2}\left(Y_{f}\right) / \mathbb{R} & =\left\{p \in L_{\#}^{2}\left(Y_{f}\right): \int_{Y_{f}} p=0\right\} \\
\mathcal{H}_{\#}\left(Y_{f}\right) & =\left\{u \in L_{\#}^{2}\left(Y_{f}\right)^{d}: u=\nabla p \text { for some } p \in H_{\#}^{1}\left(Y_{f}\right) \text { satisfying } \int_{Y_{f}} p=0\right\} .
\end{aligned}
$$

Remark 2.6 In general, a Bloch function is the product of a plane wave and a periodic function, i.e.

$$
f(x, \theta)=e^{i \theta \cdot x} g(x, \theta)
$$

The eigenvalues associated to (6) are in fact Bloch functions. Indeed,

$$
\phi_{k}(x, \theta)=e^{i \theta \cdot x} v_{k}(x, \theta)
$$

where the functions $v_{k}$ are obtained from the periodic eigenvalue problem

$$
\left\{\begin{aligned}
-(\nabla+i \theta) \cdot(\nabla+i \theta) v_{k}(\theta) & =\mu_{k}^{2}(\theta) v_{k}(\theta) & & \text { in } Y_{f} \\
(\nabla+i \theta) v_{k}(\theta) \cdot \nu & =0 & & \text { on } \partial Y_{s} \\
v_{k}(x+p, \theta) & =v_{k}(x, \theta), \quad & & p \in \mathbb{Z}^{d}
\end{aligned}\right.
$$

The diagonalization of the inviscid operator $A_{0}$ on the domain $Y_{f}^{\varepsilon}$ is now obtained using the eigenvalues and eigenfunctions of the spectral problem (6) along with an appropriate rescaling. Indeed, for each $\theta \neq 0$ and $k \in \mathbb{Z}^{d} /\{0\}$, we define

$$
\begin{aligned}
\lambda_{k}^{\varepsilon}(\theta) & =\varepsilon^{-1} \lambda_{k}(\theta), \\
\Phi_{k}^{\varepsilon}(x, \theta) & =\varepsilon^{-d / 2} \Phi_{k}\left(\frac{x}{\varepsilon}, \theta\right),
\end{aligned}
$$

where

$$
\begin{aligned}
\lambda_{k}(\theta) & =\operatorname{sgn}(k) \mu_{|k|}(\theta), \\
\Phi_{k}(x, \theta) & =\left(\begin{array}{c}
\frac{\phi_{|k|}(x, \theta)}{\sqrt{2}} \\
\frac{\operatorname{sgn}(k) \nabla \phi_{|k|}(x, \theta)}{i \mu_{|k|}(\theta) \sqrt{2}}
\end{array}\right) .
\end{aligned}
$$

Remark 2.7 When $\theta=0$, we use the same definition (7), but take $1<|k|$.

We conclude

$$
\left\{\begin{aligned}
A_{0} \Phi_{k}^{\varepsilon}(\theta) & =i \lambda_{k}^{\varepsilon}(\theta) \Phi_{k}^{\varepsilon}(\theta) & & \text { in } Y_{f}^{\varepsilon} \\
{\left[\Phi_{k}^{\varepsilon}(\theta)\right]_{2} \cdot \nu } & =0 & & \text { on } \partial Y_{s}^{\varepsilon} \\
\Phi_{k}^{\varepsilon}(x+p, \theta) & =e^{i \theta \cdot p} \Phi_{k}^{\varepsilon}(x, \theta), & & p \in \mathbb{Z}^{d} .
\end{aligned}\right.
$$

Now, for each $\theta \in B$, we define $S_{k}^{\varepsilon}(\theta)$ to be the space spanned by $\Phi_{k}^{\varepsilon}(\theta)$ and we define $\Pi_{k}^{\varepsilon}(\theta)$ to be the orthogonal projection from $L_{\theta}^{2}\left(Y_{f}^{\varepsilon}\right)^{d+1}$ onto $S_{k}^{\varepsilon}(\theta)$.

We define the following mutually orthogonal subspaces on $L^{2}\left(\mathbb{R}_{\varepsilon}^{d}\right)^{d+1}$

$$
\begin{aligned}
S_{k}^{\varepsilon} & =\left(I^{\varepsilon}\right)^{-1} f_{B} S_{k}^{\varepsilon}(\theta) d \theta \\
& =\left\{u \in L^{2}\left(\mathbb{R}_{\varepsilon}^{d}\right)^{d+1} ; u_{\#}(\cdot, \theta) \in S_{k}^{\varepsilon}(\theta) \text { for a.e. } \theta \in B\right\},
\end{aligned}
$$


where $I^{\varepsilon}$ is the mapping given in proposition 2.4. We denote $\Pi_{k}^{\varepsilon}$ as the orthogonal projection from $L^{2}\left(\mathbb{R}_{\varepsilon}^{d}\right)^{d+1}$ onto $S_{k}^{\varepsilon}$. Notice that we have

$$
\Pi_{k}^{\varepsilon} \Phi=\left(I^{\varepsilon}\right)^{-1} f_{B} \Pi_{k}^{\varepsilon}(\theta) I^{\varepsilon} \Phi d \theta
$$

As a result of Proposition 2.5, the mutually orthogonal spaces $S_{k}^{\varepsilon}(\theta)$ have the following direct sum decomposition in $L_{\theta}^{2}\left(Y_{f}^{\varepsilon}\right)^{d+1}$ :

$$
\bigoplus_{1 \leq|k|} S_{k}^{\varepsilon}(\theta)=L_{\theta}^{2}\left(Y_{f}^{\varepsilon}\right) \times \mathcal{H}_{\theta}\left(Y_{f}^{\varepsilon}\right), \quad \text { when } \theta \neq 0
$$

Integrating in $\theta$ and applying $\left(I^{\varepsilon}\right)^{-1}$ to (9), we have the following result. The proof will be given in subsection 4.2 .

Proposition 2.8

$$
\bigoplus_{1<|k|} S_{k}^{\varepsilon} \subseteq L^{2}\left(\mathbb{R}_{\varepsilon}^{d}\right) \times \mathcal{H}\left(\mathbb{R}_{\varepsilon}^{d}\right) \subseteq \bigoplus_{1 \leq k} S_{k}^{\varepsilon}=L^{2}\left(\mathbb{R}_{\varepsilon}^{d}\right) \times \mathcal{H}_{l o c}\left(\mathbb{R}_{\varepsilon}^{d}\right)
$$

where

$$
\begin{aligned}
\mathcal{H}\left(\mathbb{R}_{\varepsilon}^{d}\right) & =\left\{u \in L^{2}\left(\mathbb{R}_{\varepsilon}^{d}\right)^{d} ; u=\nabla p, \text { for } p \in H^{1}\left(\mathbb{R}_{\varepsilon}^{d}\right)\right\}, \\
\mathcal{H}_{\text {loc }}\left(\mathbb{R}_{\varepsilon}^{d}\right) & =\left\{u \in L^{2}\left(\mathbb{R}_{\varepsilon}^{d}\right)^{d} ; u=\nabla p, \text { for } p \in H_{\text {loc }}^{1}\left(\mathbb{R}_{\varepsilon}^{d}\right)\right\}
\end{aligned}
$$

Furthermore, $\left[f^{\varepsilon}\right]_{2}$ can be written as $\nabla g^{\varepsilon}$ and satisfies the inequality

$$
\left\|g^{\varepsilon}\right\|_{L^{2}\left(\mathbb{R}_{\varepsilon}^{d}\right)} \leq C \varepsilon\left\|\nabla g^{\varepsilon}\right\|_{L^{2}\left(\mathbb{R}_{\varepsilon}^{d}\right)}, \quad \text { whenever } f^{\varepsilon} \in \bigoplus_{1<|k|} S_{k}^{\varepsilon}
$$

\section{Main Results}

Here, we state our main results. The first subsection is about the modified Helmholtz operator that allows us to split the micro-incompressible and the micro-acoustic parts of the flow. The second one is about some convergence results.

\subsection{Modified Helmholtz Operators}

Using the Bloch decomposition, we introduce the modified Helmholtz operators $P_{M}^{\varepsilon}, Q_{M}^{\varepsilon}$. These operators serve as the basis for the current article, and our final paper [9]. In summary, they enable us to precisely partition the flow into an incompressible part and an acoustic one on the microscopic scale. Consequently a description of the flow in all regions of time and space is possible.

We begin by recalling the usual Helmholtz operators $P^{\varepsilon}, Q^{\varepsilon}$ acting on $L^{2}\left(\mathbb{R}_{\varepsilon}^{d}\right)^{d}$. We define them by

$$
P^{\varepsilon}=I-Q^{\varepsilon}, \quad Q^{\varepsilon}=\left(I^{\varepsilon}\right)^{-1} f_{B} Q^{\varepsilon}(\theta) d \theta I^{\varepsilon}
$$

where

$$
Q^{\varepsilon}(\theta) f=\nabla g \quad \text { for } \theta \neq 0
$$

and $g$ is defined by the system

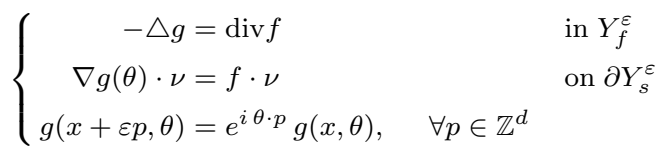


It is not difficult to see that $P^{\varepsilon} f, Q^{\varepsilon} f$ indeed yield the usual Helmholtz operators. That is,

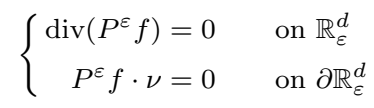

and that $Q^{\varepsilon} f$ is a gradient (see [8]).

What we find interesting is how Helmholtz operators $P^{\varepsilon}, Q^{\varepsilon}$ defined in (12) can also be characterized through the projection operators $\Pi_{k}^{\varepsilon}$. Indeed, as a consequence of direct sum (9), we have the following

$$
Q^{\varepsilon}(\theta) f(\theta)=\sum_{1 \leq|k|}\left[\Pi_{k}^{\varepsilon}(\theta)(0, f(\theta))\right]_{2}, \quad \text { when } \theta \neq 0 .
$$

Here $f(\theta)$ belongs to $L_{\theta}^{2}\left(Y_{f}^{\varepsilon}\right)^{d}$ and $(0, f(\theta)) \in \mathbb{C} \times \mathbb{C}^{d}$. It follows that

$$
Q^{\varepsilon} f=\sum_{1 \leq|k|}\left[\Pi_{k}^{\varepsilon}(0, f)\right]_{2}
$$

for $f \in L^{2}\left(\mathbb{R}_{\varepsilon}^{d}\right)^{d}$.

Remark 3.1 When $\theta=0$, we denote $P_{y}^{\varepsilon}, Q_{y}^{\varepsilon}$ the Helmholtz operators on the domain $Y_{f}^{\varepsilon}$. That is

$$
P_{y}^{\varepsilon}=I-Q_{y}^{\varepsilon}
$$

where

$$
Q_{y}^{\varepsilon} f=\nabla g
$$

and $g$ is defined by the system

$$
\left\{\begin{aligned}
-\triangle g & =\operatorname{div} f \quad \text { in } Y_{f}^{\varepsilon} \\
\nabla g \cdot \nu & =f \cdot \nu \quad \text { on } \partial Y_{s}^{\varepsilon} \\
\int_{Y_{f}^{\varepsilon}} g & =0 \\
g & \text { is } Y \text {-periodic }
\end{aligned}\right.
$$

As a result of Proposition 2.5, we have the following direct sum in $L_{\#}^{2}\left(Y_{f}^{\varepsilon}\right)^{d+1}$

$$
\bigoplus_{1<|k|} S_{k}^{\varepsilon}(0)=L_{\#}^{2}\left(Y_{f}^{\varepsilon}\right) / \mathbb{R} \times \mathcal{H}_{\#}\left(Y_{f}^{\varepsilon}\right)
$$

hence

$$
Q_{y}^{\varepsilon} f=\sum_{1<|k|}\left[\Pi_{k}^{\varepsilon}(0)(0, f)\right]_{2},
$$

where $f \in L_{\#}^{2}\left(Y_{f}^{\varepsilon}\right)^{d}$, and $(0, f)$ belongs to $\mathbb{R} \times \mathbb{R}^{d}$.

We now introduce the modified Helmholtz operators $P_{M}^{\varepsilon}, Q_{M}^{\varepsilon}$ acting on $L^{2}\left(\mathbb{R}_{\varepsilon}^{d}\right)^{d}$ we define $P_{M}^{\varepsilon}=$ $I-Q_{M}^{\varepsilon}$, where $Q_{M}^{\varepsilon}$ is given by

$$
Q_{M}^{\varepsilon} f=\sum_{1<|k|}\left[\Pi_{k}^{\varepsilon}(0, f)\right]_{2} .
$$

Note that we have the following relationship

$$
\begin{aligned}
P_{M}^{\varepsilon} f & =P^{\varepsilon} f+\left[\left(\Pi_{1}^{\varepsilon}+\Pi_{-1}^{\varepsilon}\right)(0, f)\right]_{2}, \\
Q_{M}^{\varepsilon} f & =Q^{\varepsilon} f-\left[\left(\Pi_{1}^{\varepsilon}+\Pi_{-1}^{\varepsilon}\right)(0, f)\right]_{2} .
\end{aligned}
$$


Our main results of this section are state the asymptotic properties of the quantities

$$
g_{1}^{\varepsilon}=\left[\left(\Pi_{1}^{\varepsilon}+\Pi_{-1}^{\varepsilon}\right)\left(g^{\varepsilon}, 0\right)\right]_{1}, \quad P_{M}^{\varepsilon} f^{\varepsilon}, \quad Q_{M}^{\varepsilon} f^{\varepsilon} .
$$

on the microscopic level. In order to precisely state the main results of this section, we extend functions from $\mathbb{R}_{\varepsilon}^{d}$ to all of $\mathbb{R}^{d}$ in the following way.

Definition 3.2 Let $f$ belong to $L^{2}\left(\mathbb{R}_{\varepsilon}^{d}\right)$. We extend $f$ to all of $\mathbb{R}^{d}$ in the following way

$$
\tilde{f}= \begin{cases}f(x) & \text { when } x \in \mathbb{R}_{\varepsilon}^{d} \\ f_{Y_{f, p}^{\varepsilon}}^{\varepsilon} f(y) & \text { when } x \in Y_{s, p}^{\varepsilon}, \forall p \in \mathbb{Z}^{d} \quad .\end{cases}
$$

We have the following results, proofs of which can be found in section 4 .

Theorem 3.3 Let $g^{\varepsilon}$ be a bounded sequence in $L^{2}\left(\mathbb{R}_{\varepsilon}^{d}\right)$ and suppose $\tilde{g}^{\varepsilon} \rightarrow g$ in $L^{2}\left(\mathbb{R}_{\varepsilon}^{d}\right)$. Then $g_{1}^{\varepsilon}$ two-scale converges to $g(x)$.

Theorem 3.4 Let $f^{\varepsilon}$ be a bounded sequence in $L^{2}\left(\mathbb{R}_{\varepsilon}^{d}\right)^{d}$. Let $f_{0}(x, y)$ denote the two-scale limit of $f^{\varepsilon}$. Then $P_{M}^{\varepsilon} f^{\varepsilon}, Q_{M}^{\varepsilon} f^{\varepsilon}$ two-scale converge to $P_{y} f_{0}, Q_{y} f_{0}$ where $P_{y}, Q_{y}$ are the usual Helmholtz operators on the domain $Y_{f}$ (see Remark 3.1).

Motivated by the formal asymptotic analysis for the case $\beta=2$, along with the results of Theorems $3.3,3.4$, we conclude this section with the following definitions.

Definition 3.5 Micro-incompressible flow is defined to be the solution to system (1) with initial condition $\left(b_{1}^{\varepsilon}, P_{M}^{\varepsilon} a^{\varepsilon}\right)$, where

$$
b_{1}^{\varepsilon}=\left[\left(\Pi_{-1}^{\varepsilon}+\Pi_{1}^{\varepsilon}\right)\left(b^{\varepsilon}, 0\right)\right]_{1} .
$$

We define micro-acoustic flow to be the solution to system (1) with initial condition $\left(b_{2}^{\varepsilon}, Q_{M}^{\varepsilon} a^{\varepsilon}\right)$, where

$$
b_{2}^{\varepsilon}=b^{\varepsilon}-b_{1}^{\varepsilon} .
$$

\subsection{Convergence results}

In order to precisely state all the convergence results associated to micro-incompressible flow, we need to impose the following conditions on the initial data $\left(b^{\varepsilon}, a^{\varepsilon}\right)$ :

(i) $\left(b^{\varepsilon}, a^{\varepsilon}\right)$ is a bounded sequence in $L^{2}\left(\mathbb{R}_{\varepsilon}^{d}\right)^{d+1}$ and

$$
\begin{aligned}
& \widetilde{b}^{\varepsilon} \longrightarrow b(x) \\
& a^{\varepsilon} \stackrel{2-s}{\longrightarrow} a_{0}(x, y) .
\end{aligned}
$$

(ii) We decompose $b^{\varepsilon}=b_{1}^{\varepsilon}+b_{2}^{\varepsilon}$ where

$$
\begin{aligned}
& b_{1}^{\varepsilon}=\left[\left(\Pi_{-1}^{\varepsilon}+\Pi_{1}^{\varepsilon}\right)\left(b^{\varepsilon}, 0\right)\right]_{1} \\
& b_{2}^{\varepsilon}=b^{\varepsilon}-b_{1}^{\varepsilon},
\end{aligned}
$$

and we assume that $b_{1}^{\varepsilon}$ converges strongly to $b(x)$ in $L^{2}\left(\mathbb{R}_{\varepsilon}^{d}\right)$.

(iii) $P_{y} a_{0}(x, y)$ is an admissible test function and $P_{M}^{\varepsilon} a^{\varepsilon} \stackrel{2-s}{\rightarrow} P_{y} a_{0}(x, y)$. 
Lastly, we define $\left(q^{\varepsilon}, v^{\varepsilon}\right)$ to be the solution to (1) with initial value $\left(b_{2}^{\varepsilon}, Q_{M}^{\varepsilon} a^{\varepsilon}\right)$. The main results read as follows

Theorem 3.6 $(1<\beta<2)$ Let $(\mathbf{S} 1)$ hold, and assume $T \in(0, \infty)$. Assume sufficient regularity and compatibility conditions on the initial data $\left(b(x), P_{y} a_{0}(x, y)\right)$ and let $\left(u^{\varepsilon}, p^{\varepsilon}\right)$ be the solution to $(1)$ for $1<\beta<2$. Then

$$
\begin{aligned}
& \sup _{0 \leq t \leq T}\left\|p^{\varepsilon}(t, x)-b(x)-q^{\varepsilon}(t, x)\right\|_{L^{2}\left(\mathbb{R}_{\varepsilon}^{d}\right)}^{2} \stackrel{\varepsilon \rightarrow 0}{\rightarrow 0},
\end{aligned}
$$

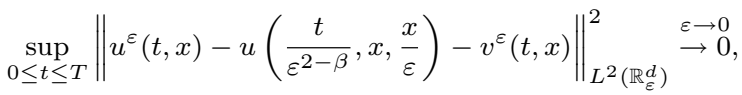

$$
\begin{aligned}
& \varepsilon^{\beta} \int_{0}^{T}\left\|\nabla u^{\varepsilon}-\frac{1}{\varepsilon} \nabla_{y} u\left(\frac{t}{\varepsilon^{2-\beta}}, x, \frac{x}{\varepsilon}\right)-\nabla v^{\varepsilon}(t, x)\right\|_{L^{2}\left(\mathbb{R}_{\varepsilon}^{d}\right)}^{2} \stackrel{\varepsilon \rightarrow 0}{\rightarrow} 0,
\end{aligned}
$$

where $(p(\tau, x, y), u(\tau, x, y))$ is the solution to the following two-scale Stokes system

$$
\left\{\begin{array}{rlrl}
\partial_{\tau} u-\triangle_{y} u+\nabla_{y} p & =0 & & \\
\operatorname{div}_{y} u & =0 & & \\
u & =0 & & \\
\int_{Y_{f}} p & =0 & & \\
u(\tau=0) & =P_{y} a_{0}(x, y) & & \\
y \rightarrow(p, u) & \text { is } Y \text {-periodic } &
\end{array}\right.
$$

Remark 3.7 The relationship between the time variables $\tau$ and $t$ is given by $\tau=t / \varepsilon^{2-\beta}$. This is due to the initial layer in time of size $\varepsilon^{2-\beta}$ that remains from the case of strongly viscous flow (see [10]). Notice that this layer in time decouples the micro-incompressible part of the flow as $\varepsilon \rightarrow 0^{+}$(see Figure 2). We also note that if $\left(b_{2}^{\varepsilon}, Q_{M}^{\varepsilon} a^{\varepsilon}\right)$ goes strongly to 0 in $L^{2}$, then $\left(q^{\varepsilon}, v^{\varepsilon}\right)$ can be replaced by 0 in the statement of the theorem.

Theorem 3.8 $(\beta=2)$ Let (S1) hold, and assume $T \in(0, \infty)$. Assume sufficient regularity on the initial data $\left(b(x), P_{y} a_{0}(x, y)\right)$ and let $\left(u^{\varepsilon}, p^{\varepsilon}\right)$ be the solution to $(1)$ for $\beta=2$. Then

$$
\begin{aligned}
& \sup _{0 \leq t \leq T}\left\|p^{\varepsilon}(t, x)-p(t, x)-q^{\varepsilon}(t, x)\right\|_{L^{2}\left(\mathbb{R}_{\varepsilon}^{d}\right)}^{2} \stackrel{\varepsilon \rightarrow 0}{\rightarrow} 0,
\end{aligned}
$$

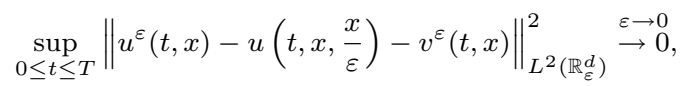

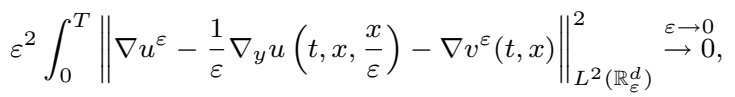

where $(p(t, x), u(t, x, y))$ is the solution to the following two-scale Stokes/Acoustic system

$$
\left\{\begin{array}{rlrl}
\left|Y_{f}\right| \partial_{t} p+\operatorname{div}_{x} \int_{Y_{f}} u & =0 & & \text { in } \mathbb{R}^{d} \\
\partial_{t} u-\triangle_{y} u+\nabla_{x} p+\nabla_{y} p_{1} & =0 & & \\
\operatorname{div}_{y} u & =0 & & \text { in } \mathbb{R}^{d} \times Y_{f} \\
u & =0 & & \text { on } \partial Y_{s} \\
\int_{Y_{f}} p_{1} & =0 & & \\
p(t=0) & =b(x) & \\
u(t=0) & =P_{y} a_{0}(x, y) & \\
y \rightarrow\left(p_{1}, u\right) & \text { is } Y \text {-periodic } &
\end{array}\right.
$$


Remark 3.9 Notice that the layer in time of size $\varepsilon^{2-\beta}$ disappears at $\beta=2$, hence the micro-incompressible part of the flow becomes coupled. In particular, we obtain the coupled macro-micro system given in (17). We refer the reader to Figure 1 for the diagram illustrating the behavior of the flow near initial time.

Theorem $3.10(2<\beta)$ Let $(\mathbf{S 1})$ hold, and assume $T \in(0, \infty)$. Assume sufficient regularity on the initial data $\left(b(x), P_{y} a_{0}(x, y)\right)$ and let $\left(u^{\varepsilon}, p^{\varepsilon}\right)$ be the solution to $(1)$ for $2<\beta$. Then

$$
\begin{aligned}
& \sup _{0 \leq t \leq T}\left\|p^{\varepsilon}(t, x)-p(t, x)-q^{\varepsilon}(t, x)\right\|_{L^{2}\left(\mathbb{R}_{\varepsilon}^{d}\right)}^{2} \stackrel{\varepsilon \rightarrow 0}{\rightarrow} 0, \\
& \sup _{0 \leq t \leq T}\left\|u^{\varepsilon}(t, x)-u\left(t, x, \frac{x}{\varepsilon}\right)-v^{\varepsilon}(t, x)\right\|_{L^{2}\left(\mathbb{R}_{\varepsilon}^{d}\right)}^{2} \stackrel{\varepsilon \rightarrow 0}{\rightarrow 0}, \\
& \varepsilon^{\beta} \int_{0}^{T}\left\|\nabla u^{\varepsilon}-\nabla v^{\varepsilon}\right\|_{L^{2}\left(\mathbb{R}_{\varepsilon}^{d}\right)}^{2} \stackrel{\varepsilon \rightarrow 0}{\rightarrow 0},
\end{aligned}
$$

where $(p(t, x), u(t, x, y))$ is the solution to the following two-scale acoustic system

$$
\left\{\begin{array}{rlrl}
\left|Y_{f}\right| \partial_{t} p+\operatorname{div} x \int_{Y_{f}} u & =0 & & \text { in } \mathbb{R}^{d} \\
\partial_{t} u+\nabla_{x} p+\nabla_{y} p_{1} & =0 & & \\
\operatorname{div}_{y} u & =0 & & \\
u \cdot \nu & =0 & & \\
\int_{Y_{f}} p_{1} & =0 & & \\
p(t=0) & =b(x) & & \\
& & & \\
u(t=0) & =Y_{s} a_{0}(x, y) & & \\
y \rightarrow\left(p_{1}, u\right) & \text { is } Y \text {-periodic } &
\end{array}\right.
$$

Remark 3.11 For $\beta>2$, while an initial layer in time is not present, we do have a boundary layer in space of size $\varepsilon^{\beta / 2}$ due to the fact that we can only impose the boundary condition

$$
u \cdot \nu=0 \text { on } \partial Y_{s} .
$$

A description of the boundary layer flow in space is necessary to conclude the overall behavior of the fluid. We refer the reader to the proof of Theorem 3.10 where we apply linearized Prandtl boundary layer expansions to conclude that the energy trapped is dissipated by heat flow.

We conclude this section with a few observations regarding the convergence results of this section.

\section{Remark 3.12}

(i) All the energy descriptions we obtained for $1<\beta$ have a macroscopic behavior, contrasting with the behavior of the flow when $\beta \leq 1$. We refer the reader to [10], where there is no macroscopic flow due to very strong viscosity.

(ii) Notice the intensity in which energy is dissipated weakens as $\beta$ increases. More precisely, notice that the micro-incompressible part of the flow only contributes to the dissipation of the energy provided $\beta \leq 2$. We show in our final paper [9] that the micro-acoustic part of the flow never dissipates the energy when $3<\beta$. That is

$$
\varepsilon^{\beta} \int_{\mathbb{R}_{\varepsilon}^{d}}\left|\nabla v^{\varepsilon}\right|^{2} \stackrel{\varepsilon \rightarrow 0}{\rightarrow 0} \quad \text { whenever } 3<\beta
$$


(iii) The oscillations of the pressure term $p^{\varepsilon}$ are completely contained in $q^{\varepsilon}$ whenever $\beta>1$.

(iv) In the three convergence Theorems for $1<\beta$, we did not try to give a rate of convergence in terms of $\varepsilon$. One can easily get some error estimate in terms of $\varepsilon$ by computing the next terms in the formal asymptotic expansions, but this is not our goal in this paper.

(v) Also, all our Theorems for $1<\beta$ have the assumption that the initial condition $\left(b(x), P_{y} a_{0}(x, y)\right)$ is sufficiently regular. We did not try to get the best possible regularity on the initial data and one can easily lower the regularity requirement on the initial data by performing a mollification in the $x$ variable. We refer the reader to [10] for details.

\section{Proof of modified Helmholtz decomposi- tion}

The main goal of this section is to prove Theorem 3.4. We start with the proof of some propositions

\subsection{Proof of proposition 2.5:}

For $\theta \in B$, we recall the following characterization

$$
\mu_{k}^{2}(\theta)=\min _{F \in \Sigma_{k}(\theta)} \max _{u \in F} \frac{\|\nabla u\|_{L^{2}\left(Y_{f}\right)}^{2}}{\|u\|_{L^{2}\left(Y_{f}\right)}^{2}}
$$

where $\Sigma_{k}(\theta)$ is the collection of all $k$-dimensional subspaces of $H_{\theta}^{1}\left(Y_{f}\right)$. Let $\xi$ belong to $B$ such that $\xi \neq \theta$. Define $F$ to be the linear span of $\left\{e^{i(\xi-\theta) \cdot x} \phi_{j}(\theta)\right\}_{j=1}^{k}$ in $H_{\xi}^{1}\left(Y_{f}\right)$ and let $u$ belong to $F$. Then

$$
\begin{aligned}
\|\nabla u\|_{L^{2}\left(Y_{f}\right)}^{2} & =\left\|\nabla_{\xi-\theta} \tilde{u}\right\|_{L^{2}\left(Y_{f}\right)}^{2} \\
& =\|\nabla \tilde{u}\|_{L^{2}\left(Y_{f}\right)}^{2}+2 \mathcal{R} e(\nabla \tilde{u}, i(\xi-\theta) \tilde{u})+|\theta-\xi|^{2}\|\tilde{u}\|_{L^{2}\left(Y_{f}\right)}^{2} \\
& \leq\left(\mu_{k}^{2}(\theta)+2 \mu_{k}(\theta)|\theta-\xi|+|\xi-\theta|^{2}\right) \times\|u\|_{L^{2}\left(Y_{f}\right)}^{2},
\end{aligned}
$$

where $\nabla_{\theta}=\nabla+i \theta$, and $\tilde{u}$ is a linear combination of $\left\{\phi_{j}(\theta)\right\}_{j=1}^{k}$. Since $F$ has dimension $k$ in $H_{\xi}^{1}\left(Y_{f}\right)$ we conclude from (19)

$$
\mu_{k}^{2}(\xi) \leq \mu_{k}^{2}(\theta)+2 \mu_{k}(\theta)|\theta-\xi|+|\xi-\theta|^{2} .
$$

Switching the roles of $\theta$ and $\xi$ allows us to conclude (i).

We now prove (ii), beginning with the proof of

$$
0 \leq \inf _{\theta \in B} \mu_{1}^{2}(\theta) \leq \inf _{\theta \in B} \mu_{2}^{2}(\theta) \leq \ldots \inf _{\theta \in B} \mu_{k}^{2}(\theta) \leq \ldots \stackrel{k \rightarrow \infty}{\rightarrow} \infty
$$

Observe that $\left\{\inf _{\theta} \mu_{k}^{2}(\theta)\right\}_{k=1}^{\infty}$ is a non-decreasing sequence. To see that its only accumulations point is at $\infty$, we let $\theta_{k}$ be the value for which $\mu_{k}^{2}(\theta)$ achieves its minimum. Then from (20) we conclude

$$
\mu_{k}(\theta) \leq \mu_{k}\left(\theta_{k}\right)+C=\inf _{\theta} \mu_{k}(\theta)+C
$$

Here $C$ is a constant independent of $k$. Passing to the limit in $\mathrm{k}$, we conclude (21). 
Now consider the max-min characterization of the principle eigenvalue

$$
\mu_{1}^{2}(\theta)=\min _{\substack{u \in H_{\theta}^{1}\left(Y_{f}\right) \\ u \neq 0}} \frac{\|\nabla u\|_{L^{2}\left(Y_{f}\right)}^{2}}{\|u\|_{L^{2}\left(Y_{f}\right)}^{2}}
$$

Taking test function $e^{i \theta \cdot x}$ belonging to $H_{\theta}^{1}\left(Y_{f}\right)$ in (22) we conclude

$$
\mu_{1}^{2}(\theta) \leq|\theta|^{2}
$$

hence

$$
\inf _{\theta \in B} \mu_{1}^{2}(\theta)=0=\mu_{1}(0)
$$

We note from system (6) that

$$
\mu_{2}^{2}(\theta)>0 \quad \text { for } \theta \in(0,2 \pi)^{d}
$$

for if $\mu_{2}^{2}(\theta)$ is zero at some $\theta \in(0,2 \pi)^{d}$, then its corresponding eigenvector must be zero due to the $\theta$-quasi periodic boundary conditions. Thus it remains to show $\mu_{2}^{2}(\theta)$ is positive for $\theta=0$. We have the variational formula

$$
\mu_{2}^{2}(0)=\min _{F \in \Sigma_{2}(0)} \max _{u \in F} \frac{\|\nabla u\|_{L^{2}\left(Y_{f}\right)}^{2}}{\|u\|_{L^{2}\left(Y_{f}\right)}^{2}}=\max _{F \in \Sigma_{1}(0)} \min _{u \in F^{\perp}} \frac{\|\nabla u\|_{L^{2}\left(Y_{f}\right)}^{2}}{\|u\|_{L^{2}\left(Y_{f}\right)}^{2}} \geq \min _{u \in H_{\#}^{1}\left(Y_{f}\right) / \mathbb{R}} \frac{\|\nabla u\|_{L_{\#}^{2}\left(Y_{f}\right)}^{2}}{\|u\|_{L_{\#}^{2}\left(Y_{f}\right)}^{2}}>0,
$$

where the inequality follows by taking the subspace $F$ to be the constants. The proof of (ii) is now complete.

For the proof of (iii), notice that (20) implies the inequalities

$$
\begin{aligned}
& \mu_{1}(\theta) \leq|\theta|, \\
& \mu_{2}(0) \leq \mu_{2}(\theta)+|\theta| .
\end{aligned}
$$

If we take the ball

$$
B_{R_{1}}(0)=\left\{\theta \in \mathbb{R}^{d}:|\theta|<R_{1}\right\}, \quad R_{1}=\mu_{2}(0) / 2,
$$

then the inequalities in (23) imply

$$
\mu_{1}(\theta)<\mu_{2}(\theta) \quad \text { on } B_{R_{1}}(0) .
$$

Hence, our claim about the simplicity of the principle eigenvalue follows.

To prove (iv) and (v), we start by explaining the construction of the $\phi_{k}$. We recall the Poincare inequality. For $\theta \in(0,2 \pi)^{d}$ there exists a constant $C(\theta)>0$ such that

$$
\|u\|_{2} \leq C(\theta)\|\nabla u\|_{2} \quad \forall u \in H_{\theta}^{1}\left(Y_{f}\right)
$$

Let's assume for the moment that (24) holds. Then given $f \in L_{\theta}^{2}\left(Y_{f}\right)$, there exists a unique $u \in$ $H_{\theta}^{1}\left(Y_{f}\right)$ satisfying

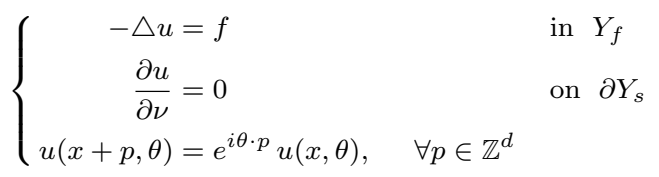

i.e.

$$
\int_{Y_{f}} \nabla u(x, \theta) \cdot \nabla \sigma(x, \theta)^{*} d x=\int_{Y_{f}} f(x) \sigma(x, \theta)^{*} d x \quad \forall \sigma \in\left(H_{\theta}^{1}\left(Y_{f}\right),\|\nabla \cdot\|_{2}\right) .
$$


Now, we define the mapping

$$
L_{\theta}^{2}\left(Y_{f}\right) \ni f \stackrel{K_{\theta}}{\longrightarrow} u \in L_{\theta}^{2}\left(Y_{f}\right) .
$$

The mapping $K_{\theta}$ is linear and continuous. Using (25) we can easily deduce the self-adjointness of $K_{\theta} . K_{\theta}$ is also a compact operator as a consequence of the Poincare inequality (24) and Sobolev compactness. It follows from Hilbert-Schmidt theorem that there exist $\phi_{k}(\theta), \mu_{k}^{2}(\theta), k=1,2,3, \ldots$ satisfying (6) such that the functions $\phi_{k}(\theta)$ form an orthonormal basis of $L_{\theta}^{2}\left(Y_{f}\right)$. Using the weak formulation of the Neumann problem (6) we conclude $\left\{\frac{\left.\nabla \phi_{k}(\theta)\right\}_{k}}{\mu_{k}(\theta)}\right\}_{k=1}^{\infty}$ forms an orthonormal basis of $\left(\mathcal{H}_{\theta}^{1}\left(Y_{f}\right),\|\nabla \cdot\|_{2}\right)$.

When $\theta=0$, we have the classical periodic case. We note that the principle eigenvalue is zero, and its corresponding eigenvector is a constant. Then to obtain the remaining eigenvectors and eigenvalues of (6), we look at the space $L_{\#}^{2}\left(Y_{f}\right) / \mathbb{R}$ and we argue as above.

We now prove (24). For this we appeal to the closed graph theorem. We first remark

$$
\left(H_{\theta}^{1}\left(Y_{f}\right),\|\nabla \cdot\|_{2}\right)
$$

is a Banach space for each $\theta \in(0,2 \pi)^{d}$. Indeed, what is required to show is the completeness, since it is a normed space. Let $u_{m}$ be a Cauchy sequence in (26). Then there exists $v \in L_{\theta}^{2}\left(Y_{f}\right)$ such that $\nabla u_{m} \rightarrow v$ in $L^{2}\left(Y_{f}\right)$. To see $v$ is a gradient we appeal to the following well known lemma, a proof of which can be found in [26] (see Remark 1.9).

Lemma 4.1 Let $\theta \in[0,2 \pi)^{d}$ and $f(\theta)$ belong to $L_{\theta}^{2}\left(Y_{f}^{\varepsilon}\right)^{d}$. Suppose that

$$
\int_{Y_{f}^{\varepsilon}} f(\theta, x) \cdot \sigma(\theta, x)^{*} d x=0
$$

for all $\sigma(\theta) \in L_{\theta}^{2}\left(Y_{f}\right)^{d}$ satisfying

$$
\begin{cases}\operatorname{div} \sigma=0 & \text { in } Y_{f}^{\varepsilon} \\ \sigma \cdot \nu=0 & \text { on } \partial Y_{s}^{\varepsilon} .\end{cases}
$$

Then there exists $g(\theta) \in H_{\theta}^{1}\left(Y_{f}^{\varepsilon}\right)$ such that $f(\theta)=\nabla g(\theta)$.

Now take $\sigma \in L_{\theta}^{2}\left(Y_{f}\right)^{d}$, divergence free in $x$ and $\theta$-quasi periodic. Then

$$
\int_{Y_{f}} v \cdot \sigma^{*}=\lim _{m \rightarrow \infty} \int_{Y_{f}} \nabla u_{m} \cdot \sigma^{*}=0
$$

It follows that $v=\nabla p$ for some $p \in H_{\theta}^{1}\left(Y_{f}\right)$. Since the space defined in (26) is a Banach space, one can apply the closed graph theorem to the identity mapping

$$
i:\left(H_{\theta}^{1}\left(Y_{f}\right),\left(\|\cdot\|_{2}^{2}+\|\nabla \cdot\|_{2}^{2}\right)^{1 / 2}\right) \rightarrow\left(H_{\theta}^{1}\left(Y_{f}\right),\|\nabla \cdot\|_{2}\right)
$$

to conclude that $i^{-1}$ is continuous, hence (24) follows and the proof of Proposition 2.5 is now complete. 


\subsection{Proof of Proposition 2.8:}

We begin with the proof of the first inclusion in (10) and inequality (11). Assume $f$ belongs to $\bigoplus_{1<|k|} S_{k}^{\varepsilon}$; we need to show that $[f]_{2}$ belongs to $\mathcal{H}\left(\mathbb{R}_{\varepsilon}^{d}\right)$. Notice that

$$
f_{B}\left[\Phi_{k}^{\varepsilon}(\theta)\right]_{2} d \theta=f_{B} \frac{\operatorname{sgn}(k) \nabla \phi_{|k|}(\theta)}{i \mu_{|k|}(\theta) \sqrt{2}} d \theta=\nabla f_{B} \frac{\operatorname{sgn}(k) \phi_{|k|}(\theta)}{i \mu_{|k|}(\theta) \sqrt{2}} d \theta
$$

whenever $1<|k|$ (see remark 4.3 for the case $|k|=1$ ). We therefore have the following expansion

$$
[f]_{2}=\sum_{1<|k|}\left[\Pi_{k}^{\varepsilon} f\right]_{2}=\sum_{1<|k|} \nabla g_{k}^{\varepsilon}
$$

where

$$
\begin{aligned}
g_{k}^{\varepsilon} & =\varepsilon f_{B} B_{k}^{\varepsilon}(\theta) \frac{\operatorname{sgn}(k)\left[\Phi_{k}^{\varepsilon}\right]_{1}(\theta)}{i \mu_{|k|}(\theta)} d \theta, \\
B_{k}^{\varepsilon}(\theta) & =\left(f_{\#}(\theta), \Phi_{k}^{\varepsilon}(\theta)\right)_{L^{2}\left(Y_{f}\right)} .
\end{aligned}
$$

It follows that $\left[f^{\varepsilon}\right]_{2}=\nabla g^{\varepsilon}$, where $g^{\varepsilon}=\sum_{1<|k|} g_{k}^{\varepsilon}$. Moreover, we have the bounds

$$
\int_{\mathbb{R}_{\varepsilon}^{d}}\left|g_{k}^{\varepsilon}\right|^{2} \leq \frac{C \varepsilon}{\inf _{\theta} \mu_{2}^{2}(\theta)} f_{B}\left|B_{k}^{\varepsilon}\right|^{2}=\frac{C \varepsilon}{\inf _{\theta} \mu_{2}^{2}(\theta)} \int_{\mathbb{R}_{\varepsilon}^{d}}\left|\left[\Pi_{k}^{\varepsilon} f\right]_{2}\right|^{2} .
$$

We deduce the inequality in (11) by summing (30) over $k$. The proof of the first inclusion is now complete. We now prove the equality in (10) beginning with

$$
\bigoplus_{1 \leq|k|} S_{k}^{\varepsilon} \subseteq L^{2}\left(\mathbb{R}_{\varepsilon}^{d}\right) \times \mathcal{H}_{l o c}\left(\mathbb{R}_{\varepsilon}^{d}\right)
$$

Suppose $f$ belongs to the left hand side of (31), it suffices to show $\left[\Pi_{ \pm 1}^{\varepsilon} f\right]_{2} \in \mathcal{H}_{\text {loc }}\left(\mathbb{R}_{\varepsilon}^{d}\right)$. We have the following classical lemma which characterizes the space $\mathcal{H}_{\text {loc }}\left(\mathbb{R}_{\varepsilon}^{d}\right)$ :

Lemma $4.2 f \in L^{2}\left(\mathbb{R}_{\varepsilon}^{d}\right)^{d}$ belongs to $\mathcal{H}_{l o c}\left(\mathbb{R}_{\varepsilon}^{d}\right)$ if and only if

$$
\int_{\mathbb{R}_{\varepsilon}^{d}} f \cdot \psi=0
$$

for all $\psi \in H^{1}\left(\mathbb{R}_{\varepsilon}^{d}\right)^{d}$ which satisfy $\operatorname{div} \psi=0$ and $\psi \cdot n=0$ on $\partial \mathbb{R}_{\varepsilon}^{d}$.

Consider the integral

$$
\int_{\mathbb{R}_{\varepsilon}^{d}}\left[\Pi_{ \pm 1}^{\varepsilon} f\right]_{2} \cdot \psi
$$

where $\psi \in H^{1}\left(\mathbb{R}_{\varepsilon}^{d}\right)^{d}$ and $\operatorname{div} \psi=0, \psi \cdot n=0$ on $\partial \mathbb{R}_{\varepsilon}^{d}$. We have

$$
\int_{\mathbb{R}_{\varepsilon}^{d}}\left[\Pi_{ \pm 1}^{\varepsilon} f\right]_{2} \cdot \psi=f_{B} \int_{Y_{f}^{\varepsilon}} B_{ \pm 1}^{\varepsilon}(\theta)\left[\Phi_{ \pm 1}^{\varepsilon}\right]_{2}(x, \theta) \cdot \psi_{\#}(x, \theta)=0
$$

where $B_{ \pm 1}^{\varepsilon}(\theta)$ is defined in (29). The proof of (31) now follows from lemma 4.2. 
To prove the other inclusion, namely

$$
\bigoplus_{1 \leq|k|} S_{k}^{\varepsilon} \supseteq L^{2}\left(\mathbb{R}_{\varepsilon}^{d}\right) \times \mathcal{H}_{l o c}\left(\mathbb{R}_{\varepsilon}^{d}\right)
$$

we take $f$ belonging to the right hand side of (32). As a consequence of Lemma 4.2 we have the following

$$
f_{B} \int_{Y_{f}^{\varepsilon}}\left[f_{\#}\right]_{2}(x, \theta) \cdot \psi_{\#}(x, \theta)=0
$$

for all $\psi_{\#}$ belonging to $f_{B} H_{\theta 0}^{1}\left(Y_{f}^{\varepsilon}\right) d \theta$ and satisfying $\operatorname{div} \psi_{\#}=0$. It follows that $\left[f_{\#}(\theta)\right]_{2}$ belongs to $H_{\theta}\left(Y_{f}^{\varepsilon}\right)$ and as a result of Proposition 2.5 we have

$$
\left[f_{\#}\right]_{2}(\theta)=\sum_{1 \leq|k|}\left[\Pi_{k}^{\varepsilon}(\theta) f\right]_{2}
$$

The proof of proposition 2.8 is now complete.

Remark 4.3 In fact, we have the strict inclusion

$$
L^{2}\left(\mathbb{R}_{\varepsilon}^{d}\right) \times \mathcal{H}\left(\mathbb{R}_{\varepsilon}^{d}\right) \subset \bigoplus_{1 \leq|k|} S_{k}^{\varepsilon}
$$

This follows from the fact that (28) does not hold for $|k|=1$. The failure of $\nabla$ to commute with the integral $f_{B}$ is due to the singularity that $\phi_{1}(\theta) / \mu_{1}(\theta)$ has at the origin. In particular, we have

$$
\left\|f_{B} \frac{\phi_{1}(\theta)}{\mu_{1}(\theta)} d \theta\right\|_{L^{2}\left(\mathbb{R}_{\varepsilon}^{d}\right)}=\left\|\frac{1}{\mu_{1}}\right\|_{L^{2}(B)}=\infty
$$

\subsection{Proof of theorem 3.3:}

We begin with the following relationship between the weak limits of $f^{\varepsilon}$ and $\tilde{f}^{\varepsilon}$. A proof can be found in [19].

Proposition 4.4 Let $f^{\varepsilon} \in L^{2}\left(\mathbb{R}_{\varepsilon}^{d}\right)$ be a bounded sequence. Then the following statements are equivalent.

(i) $f^{\varepsilon} \rightarrow\left|Y_{f}\right| f \quad$ in $L^{2}\left(\mathbb{R}_{\varepsilon}^{d}\right)$.

(ii) $\tilde{f}^{\varepsilon} \rightarrow f$ in $L^{2}\left(\mathbb{R}^{d}\right)$.

We now define

$$
\begin{aligned}
& g_{1}^{\varepsilon}=\left[\left(\Pi_{1}^{\varepsilon}+\Pi_{-1}^{\varepsilon}\right)\left(g^{\varepsilon}, 0\right)\right]_{1}, \\
& g_{2}^{\varepsilon}=g^{\varepsilon}-g_{1}^{\varepsilon},
\end{aligned}
$$

and we note that

$$
g_{2}^{\varepsilon}=\left[\sum_{1<|k|} \Pi_{k}^{\varepsilon}\left(g^{\varepsilon}, 0\right)\right]_{1} .
$$

We show that $g_{2}^{\varepsilon} \rightarrow 0$. Using the definitions of the projections $\Pi_{k}^{\varepsilon}$, we have the equality

$$
\int_{\mathbb{R}_{\varepsilon}^{d}} g_{2}^{\varepsilon}(x) \sigma(x) d x=\sum_{1<|k|}^{\infty} f_{B}\left(g_{\#}^{\varepsilon}(\theta),\left[\Phi_{k}^{\varepsilon}(\theta)\right]_{1}\right)_{L^{2}\left(Y_{f}\right)}\left(\sigma_{\#}(\theta),\left[\Phi_{k}^{\varepsilon}(\theta)\right]_{1}\right)_{L^{2}\left(Y_{f}\right)}
$$


Performing an integration by parts yields

$$
\left(\sigma_{\#}(\theta),\left[\Phi_{k}^{\varepsilon}(\theta)\right]_{1}\right)_{L^{2}\left(Y_{f}\right)}=\frac{-i \varepsilon \operatorname{sgn}(k)}{\mu_{|k|}(\theta)}\left(\nabla \sigma_{\#},\left[\Phi_{k}^{\varepsilon}(\theta)\right]_{2}\right)_{L^{2}\left(Y_{f}\right)} .
$$

Hence,

$$
\begin{aligned}
\left|\int_{\mathbb{R}_{\varepsilon}^{d}} g_{2}^{\varepsilon} \sigma\right| & \leq \frac{C \varepsilon}{\inf _{\theta} \mu_{2}} \sum_{1<|k|}^{\infty}\left(f_{B}\left|\left(g_{\#}^{\varepsilon}(\theta),\left[\Phi_{k}^{\varepsilon}(\theta)\right]_{1}\right)_{L^{2}\left(Y_{f}\right)}\right|^{2}+\left|\left(\nabla \sigma_{\#}(\theta),\left[\Phi_{k}^{\varepsilon}(\theta)\right]_{2}\right)\right|^{2}\right) \\
& \leq \frac{C \varepsilon}{\inf _{\theta} \mu_{2}}\left(\left\|g^{\varepsilon}\right\|_{L^{2}\left(\mathbb{R}_{\varepsilon}^{d}\right)}^{2}+\|\nabla \sigma\|_{L^{2}\left(\mathbb{R}_{\varepsilon}^{d}\right)}^{2}\right),
\end{aligned}
$$

where the last equality follows from proposition 2.8. Sending $\varepsilon \rightarrow 0$ we conclude $g_{1}^{\varepsilon} \rightarrow\left|Y_{f}\right| g$ in $L^{2}\left(\mathbb{R}_{\varepsilon}^{d}\right)$.

To improve this weak convergence, we let $g_{0}(x, y)$ denote the two-scale limit of $g_{1}^{\varepsilon}$. It suffices to show $g_{0}(x, y)$ is independent of $y$ in $Y_{f}$. Using the definition of the projections, we write $g_{1}^{\varepsilon}$ explicitly

$$
g_{1}^{\varepsilon}(x)=2 f_{B} B_{1}^{\varepsilon}(\theta)\left[\Phi_{1}^{\varepsilon}(x, \theta)\right]_{1} d \theta
$$

where

$$
B_{1}^{\varepsilon}(\theta)=\left(g_{\#}^{\varepsilon}(\theta),\left[\Phi_{1}^{\varepsilon}(\theta)\right]_{1}\right)_{L^{2}\left(Y_{f}^{\varepsilon}\right)}
$$

Applying the gradient to $g_{1}^{\varepsilon}$ we deduce

$$
\begin{aligned}
\varepsilon \nabla g_{1}^{\varepsilon}(x) & =2 i \int_{B} B_{1}^{\varepsilon}(\theta) \mu_{1}(\theta)\left[\Phi_{1}^{\varepsilon}\right]_{2}(x, \theta) d \theta \\
& =2 i \sum_{p \in \mathbb{Z}^{d}} \hat{\mu}_{1}(p) h^{\varepsilon}(x+\varepsilon p)
\end{aligned}
$$

where we have expanded the principle eigenvalue $\mu_{1}(\theta)$ into its Fourier series, and $h^{\varepsilon}$ is defined to be

$$
h^{\varepsilon}(x)=2 f_{B} B_{1}^{\varepsilon}(\theta)\left[\Phi_{1}^{\varepsilon}\right]_{2}(x, \theta) d \theta
$$

We are now in a position to compute the two-scale limit of $\varepsilon \nabla g_{1}^{\varepsilon}$. First observe that, if we denote the two-scale limit of $h^{\varepsilon}(x)$ by $h_{0}(x, y)$, then the two-scale limit of $h^{\varepsilon}(x+\varepsilon p)$ also remains $h_{0}(x, y)$. Therefore, using equality (34), we conclude that

$$
\varepsilon \nabla g_{1}^{\varepsilon}(x) \stackrel{2-s}{\longrightarrow} 2 \sum_{p \in \mathbb{Z}^{d}} \hat{\mu}_{1}(p) h_{0}(x, y)=2 \mu_{1}(0) h_{0}(x, y)=0
$$

Now let $\sigma(x, y)$ be a test function belonging to $D\left(\mathbb{R}^{d} ; C_{\#}^{\infty}(Y)\right)^{d}$, and satisfying $\sigma(x, y)=0$ on $Y_{s}$. Then an integration by parts yields the formula

$$
-\int_{\mathbb{R}_{\varepsilon}^{d}} \varepsilon \nabla g_{1}^{\varepsilon} \cdot \sigma\left(x, \frac{x}{\varepsilon}\right)=\int_{\mathbb{R}_{\varepsilon}^{d}} \varepsilon g^{\varepsilon}\left[\operatorname{div}_{x} \sigma\left(x, \frac{x}{\varepsilon}\right)+\frac{1}{\varepsilon} \operatorname{div}_{y} \sigma\left(x, \frac{x}{\varepsilon}\right)\right] .
$$

Passing to the limit yields

$$
0=\int_{\mathbb{R}^{d}} \int_{Y_{f}} g_{0}(x, y) \operatorname{div}_{y} \sigma(x, y)
$$

hence the proof of theorem 3.3 is now complete.

Remark 4.5 As a consequence of theorem 3.3 we deduce that

$$
g_{2}^{\varepsilon} \stackrel{2-s}{\longrightarrow} g(x, y)-g(x)
$$

where $g(x, y)$ is the two-scale limit of $g^{\varepsilon}$. Furthermore, notice that all oscillations of the frequency $\varepsilon^{-1}$ associated to the sequence $g^{\varepsilon}$ are contained in $g_{2}^{\varepsilon}$. 


\subsection{Proof of theorem 3.4:}

We note that

$$
\begin{aligned}
P_{M}^{\varepsilon} f^{\varepsilon} & =P f^{\varepsilon}+\left[\left(\Pi_{1}^{\varepsilon}+\Pi_{-1}^{\varepsilon}\right)\left(0, f^{\varepsilon}\right)\right]_{2} \\
& =P f^{\varepsilon}+f_{2}^{\varepsilon}
\end{aligned}
$$

and we compute the two-scale limits of $P^{\varepsilon} f^{\varepsilon}$ and $f_{2}^{\varepsilon}$.

We begin with $P^{\varepsilon} f^{\varepsilon}$, and denote its two-scale limit by $\chi_{0}$. For $\sigma \in D\left[\mathbb{R}^{d} ; C_{\#}^{\infty}(Y)\right]^{d}$ we have the equality

$$
0=-\int_{\mathbb{R}_{\varepsilon}^{d}} \operatorname{div}\left(P f^{\varepsilon}\right) \sigma\left(x, \frac{x}{\varepsilon}\right)=\int_{\mathbb{R}_{\varepsilon}^{d}} P f^{\varepsilon} \cdot\left[\nabla_{x} \sigma\left(x, \frac{x}{\varepsilon}\right)+\frac{1}{\varepsilon} \nabla_{y} \sigma\left(x, \frac{x}{\varepsilon}\right)\right] .
$$

If we take $\sigma$ independent of $y$ and pass to the limit in (35), we obtain

$$
\int_{\mathbb{R}^{d} \times Y_{f}} \chi_{0} \cdot \nabla \sigma(x)=0 .
$$

On the other hand from equality (35), we must have

$$
\int_{\mathbb{R}_{\varepsilon}^{d}} P f^{\varepsilon} \cdot \nabla_{y} \sigma\left(x, \frac{x}{\varepsilon}\right) \stackrel{\varepsilon \rightarrow 0}{\rightarrow 0},
$$

and hence

$$
\int_{\mathbb{R}^{d} \times Y_{f}} \chi_{0} \cdot \nabla_{y} \sigma(x, y)=0 .
$$

Therefore, $\chi_{0}$ satisfies the following two-scale system

$$
\left\{\begin{aligned}
& \operatorname{div}_{x} \int_{Y_{f}} \chi_{0}=0 \text { in } \mathbb{R}^{d} \\
& \operatorname{div}_{y} \chi_{0}=0 \text { in } Y_{f} \\
& \chi_{0} \cdot \nu=0 \text { on } \partial Y_{s} \\
& y \rightarrow \chi_{0} \text { is } Y \text {-periodic }
\end{aligned}\right.
$$

We now study the limit of $f_{2}^{\varepsilon}$, and denote its two-scale limit by $\chi_{1}$. Making use of the definition of the projections mappings $\Pi_{ \pm 1}^{\varepsilon}$, we obtain

$$
f_{2}^{\varepsilon}(x)=2 f_{B} B^{\varepsilon}(\theta)\left[\Phi_{1}^{\varepsilon}(x, \theta)\right]_{2}
$$

where

$$
B^{\varepsilon}(\theta)=\left(f_{\#}^{\varepsilon}(\theta),\left[\Phi_{1}^{\varepsilon}(\theta)\right]_{2}\right)_{L^{2}\left(Y_{f}^{\varepsilon}\right)} .
$$

Taking the divergence of (37) yields

$$
\begin{aligned}
\varepsilon \operatorname{div} f_{2}^{\varepsilon}(x) & =\frac{2}{i} f_{B} \mu_{1}(\theta) B^{\varepsilon}(\theta)\left[\Phi_{1}^{\varepsilon}(x, \theta)\right]_{1} \\
& =\frac{2}{i} \int_{B} \sum_{p \in \mathbb{Z}^{d}} \widehat{\mu_{1}}(p) e^{i \theta \cdot p} B^{\varepsilon}(\theta)\left[\Phi_{1}^{\varepsilon}(x, \theta)\right]_{1} \\
& =\frac{2}{i} \sum_{p \in \mathbb{Z}^{d}} \widehat{\mu_{1}}(p) h^{\varepsilon}(x+\varepsilon p),
\end{aligned}
$$


where the last equality in (38) made use of the quasi-periodicity of $\left[\Phi_{1}^{\varepsilon}\right]_{1}$. Here $h^{\varepsilon}$ is defined as

$$
h^{\varepsilon}(x)=f_{B} B^{\varepsilon}(\theta)\left[\Phi_{1}^{\varepsilon}(x, \theta)\right]_{1} .
$$

We now pass to the limit in (38), and we begin with the left-hand side. Note that $f_{2}^{\varepsilon}$ has a normal component which vanishes on $\partial \mathbb{R}_{\varepsilon}^{d}$, hence for $\sigma \in D\left[\mathbb{R}^{d} ; C_{\#}^{\infty}(Y)\right]$ we have

$$
\begin{aligned}
& -\varepsilon \int_{\mathbb{R}_{\varepsilon}^{d}} \operatorname{div} f_{2}^{\varepsilon} \sigma\left(x, \frac{x}{\varepsilon}\right) \\
& =\int_{\mathbb{R}_{\varepsilon}^{d}} f_{2}^{\varepsilon} \cdot\left[\varepsilon \nabla_{x} \sigma\left(x, \frac{x}{\varepsilon}\right)+\nabla_{y} \sigma\left(x, \frac{x}{\varepsilon}\right)\right] \\
& \varepsilon \rightarrow 0 \\
& \stackrel{\rightarrow}{\rightarrow} \int_{\mathbb{R}^{d} \times Y_{f}} \chi_{1} \cdot \nabla_{y} \sigma .
\end{aligned}
$$

In order to pass to the limit in the right hand side of (38), we first notice that if we denote the two-scale limit of $h^{\varepsilon}$ by $h$, we have

$$
h^{\varepsilon}(x+\varepsilon p) \stackrel{2-s}{\longrightarrow} h, \quad \forall p \in \mathbb{Z}^{d} .
$$

Indeed, from a change of variables we have the equality

$$
\int_{\mathbb{R}_{\varepsilon}^{d}} h^{\varepsilon}(x+\varepsilon p) \sigma\left(x, \frac{x}{\varepsilon}\right) d x=\int_{\mathbb{R}_{\varepsilon}^{d}} h^{\varepsilon}(x) \sigma\left(x-\varepsilon p, \frac{x}{\varepsilon}\right) d x
$$

and passing to the limit in $\varepsilon$ gives us (39). It follows that

$$
\begin{aligned}
& \frac{2}{i} \sum_{p \in \mathbb{Z}^{d}} \widehat{\mu_{1}}(p) \int_{\mathbb{R}_{\varepsilon}^{d}} h^{\varepsilon}(x+\varepsilon p) \sigma\left(x, \frac{x}{\varepsilon}\right) \\
& \stackrel{\varepsilon \rightarrow 0}{\rightarrow} \frac{2}{i} \sum_{p \in \mathbb{Z}^{d}} \widehat{\mu_{1}}(p) \int_{\mathbb{R}^{d} \times Y_{f}} h \sigma \\
& =\frac{2}{i} \mu_{1}(0) \int_{\mathbb{R}^{d} \times Y_{f}} h \sigma .
\end{aligned}
$$

Since $\mu_{1}(0)=0$, we deduce the following two-scale system for $\chi_{1}$

$$
\left\{\begin{array}{c}
\operatorname{div}_{y} \chi_{1}=0 \quad \text { in } Y_{f} \\
\chi_{1} \cdot \nu=0 \quad \text { on } \partial Y_{s} \\
y \rightarrow \chi_{1} \quad \text { is } Y \text {-periodic }
\end{array}\right.
$$

To compute the two-scale limit of $Q_{M}^{\varepsilon} f^{\varepsilon}$, which we denote by $\chi_{2}$, we recall from proposition 2.8 that $Q_{M}^{\varepsilon} f^{\varepsilon}=\nabla g^{\varepsilon}$ and satisfies the inequality

$$
\left\|g^{\varepsilon}\right\|_{2} \leq C \varepsilon\left\|\nabla g^{\varepsilon}\right\|_{2} .
$$

For any $\sigma \in D\left[\mathbb{R}^{d} ; C_{\#}^{\infty}(Y)\right]^{d}$ satisfying $\sigma=0$ on $Y_{s}$ and $\operatorname{div}_{y} \sigma=0$ we have

$$
-\int_{\mathbb{R}_{\varepsilon}^{d}} Q_{M}^{\varepsilon} f^{\varepsilon} \cdot \sigma\left(x, \frac{x}{\varepsilon}\right)=\int_{\mathbb{R}_{\varepsilon}^{d}} g^{\varepsilon} \operatorname{div}_{x} \sigma\left(x, \frac{x}{\varepsilon}\right) .
$$


Using (41), we pass to the limit in (42) and deduce

$$
\int_{\mathbb{R}^{d} \times Y_{f}} \chi_{2} \cdot \sigma(x, y)=0 .
$$

The functions that are orthogonal to divergence free functions are exactly gradients. We deduce that there exist a unique function $g_{0} \in L^{2}\left(\mathbb{R}^{d} ; H_{\#}\left(Y_{f}\right) / \mathbb{R}\right)$ such that

$$
\chi_{2}=\nabla g_{0} .
$$

If we denote the two-scale limit of $f^{\varepsilon}$ by $f_{0}$, then combining all the two-scale limits above we have

$$
f_{0}=\chi_{0}+\chi_{1}+\chi_{2} .
$$

Since the decomposition

$$
f_{0}=P_{y} f_{0}+Q_{y} f_{0}
$$

is unique the proof of theorem 3.4 is complete.

Remark 4.6 As noted in the proof of theorem 3.4, we have the equality $P_{y} a_{0}=\chi_{0}+\chi_{1}$ where $\chi_{0}$ and $\chi_{1}$ satisfy $(36),(40)$ respectively. We can say slightly more about $\chi_{1}$. Indeed, notice that the

$$
\operatorname{curl}\left[\left(\Pi_{1}^{\varepsilon}+\Pi_{-1}^{\varepsilon}\right)\left(0, a^{\varepsilon}\right)\right]_{2}=0,
$$

hence

$$
\begin{aligned}
\operatorname{curl}_{x} f_{Y_{f}} \chi_{1} & =0 \\
\operatorname{curl}_{y} \chi_{1} & =0
\end{aligned}
$$

By elliptic regularity, it follows that $\chi_{1}$ is regular in $y$.

In fact, more can be said about $\chi_{1}$ in dimension $d=2$. We define

$$
V=\left\{u \in L_{\#}^{2}\left(Y_{f}\right)^{d} ; \operatorname{div} u=0 \text { on } Y_{f}, u \cdot \nu=0 \text { on } \partial Y_{s}\right\}
$$

and $\Gamma=$ curl. Then the kernel of $\Gamma$ is a Banach space of dimension $N+1$ where $N$ equals the number of holes in $Y_{f}$. We refer the reader to [17] for the details.

\section{Proof of the convergence Results}

The existence of a solution $\left(p^{\varepsilon}, u^{\varepsilon}\right) \in C\left([0, T) ; L^{2}\left(\mathbb{R}_{\varepsilon}^{d}\right) \times L^{2}\left(\mathbb{R}_{\varepsilon}^{d}\right)^{d}\right)$ to the linear system (1) is very classical and we do not recall it here. We only observe that the solution $\left(p^{\varepsilon}, u^{\varepsilon}\right)$ satisfies

$$
\left\|p^{\varepsilon}(t)\right\|_{L^{2}\left(\mathbb{R}_{\varepsilon}^{d}\right)}^{2}+\left\|u^{\varepsilon}(t)\right\|_{L^{2}\left(\mathbb{R}_{\varepsilon}^{d}\right)}^{2}+2 \varepsilon^{\beta} \int_{0}^{t}\left\|\nabla u^{\varepsilon}\right\|_{L^{2}\left(\mathbb{R}_{\varepsilon}^{d}\right)}^{2}=\left\|b^{\varepsilon}\right\|_{L^{2}\left(\mathbb{R}_{\varepsilon}^{d}\right)}^{2}+\left\|a^{\varepsilon}\right\|_{L^{2}\left(\mathbb{R}_{\varepsilon}^{d}\right)}^{2} .
$$

We note that the proofs of the main results repeatedly make use of the Poincaré inequality and the energy estimate satisfied by the sequence of solutions $\left(p^{\varepsilon}, u^{\varepsilon}\right)$. We state the Poincaré inequality on the domain $\mathbb{R}_{\varepsilon}^{d}($ see $[24])$

Theorem 5.1 Let $f \in H_{0}^{1}\left(\mathbb{R}_{\varepsilon}^{d}\right)$. Then we have

$$
\|f\|_{L^{2}\left(\mathbb{R}_{\varepsilon}^{d}\right)} \leq C \varepsilon\|\nabla f\|_{L^{2}\left(\mathbb{R}_{\varepsilon}^{d}\right)} .
$$




\section{1 $(1<\beta<2)$ Proof of theorem 3.6:}

We introduce the following error terms

$$
\begin{aligned}
& \alpha_{l}^{\varepsilon}=p^{\varepsilon}-b(x)-\left(\sum_{j=0}^{l} \varepsilon^{(2 j+1)(\beta-1)} p_{j}\left(\frac{t}{\varepsilon^{2-\beta}}, x, \frac{x}{\varepsilon}\right)\right)-q^{\varepsilon} \\
& \beta_{l}^{\varepsilon}=u^{\varepsilon}-\left(\sum_{j=0}^{l} \varepsilon^{2 j(\beta-1)} u_{j}\left(\frac{t}{\varepsilon^{2-\beta}}, x, \frac{x}{\varepsilon}\right)\right)-v^{\varepsilon}
\end{aligned}
$$

where $\left(p_{0}, u_{0}\right)=(p, u)$ is the unique solution to $(16)$, and $\left(p_{j}, u_{j}\right), j \geq 1$, satisfy the coupled two-scale system

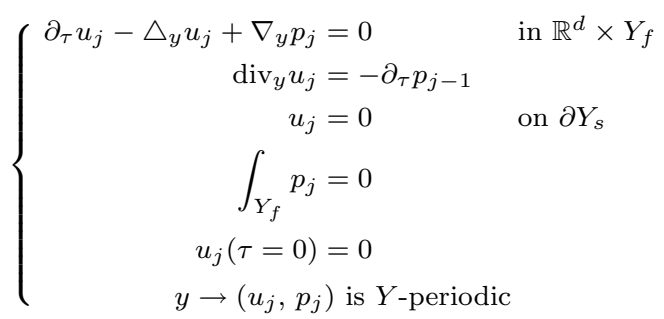

We present an analysis of the error term (43) for $l=0$ which is enough if $\beta \in(4 / 3,2)$. At the end of the subsection, we will conclude with a discussion on the adjustments needed to justify the error terms in the case $0<l$ for $\beta \in(1,4 / 3]$. For $l=0$ the proposed error term $\left(\alpha_{0}^{\varepsilon}, \beta_{0}^{\varepsilon}\right)=\left(\alpha^{\varepsilon}, \beta^{\varepsilon}\right)$ satisfies the following initial/boundary value problem

$$
\left\{\begin{aligned}
\partial_{t} \alpha^{\varepsilon}+\operatorname{div} \beta^{\varepsilon} & =F^{\varepsilon}(t, x) & & \text { in }(0, \infty) \times \mathbb{R}_{\varepsilon}^{d} \\
\partial_{t} \beta^{\varepsilon}-\varepsilon^{\beta} \triangle \beta^{\varepsilon}+\nabla \alpha^{\varepsilon} & =G^{\varepsilon}(t, x) & & \\
\beta^{\varepsilon} & =0 & & \text { on } \partial \mathbb{R}_{\varepsilon}^{d} \\
\alpha^{\varepsilon}(t=0) & =b_{1}^{\varepsilon}-b(x)-\varepsilon^{\beta-1} p_{0}(\tau=0) & & \\
\beta^{\varepsilon}(t=0) & =P_{M}^{\varepsilon} a^{\varepsilon}(x)-P_{y} a_{0}\left(x, \frac{x}{\varepsilon}\right) & &
\end{aligned}\right.
$$

where the right-hand side terms are

$$
\begin{aligned}
& F^{\varepsilon}(t, x)=-\varepsilon^{2 \beta-3} \partial_{\tau} p-\operatorname{div}_{x} u \\
& G^{\varepsilon}(t, x)=\varepsilon^{\beta} \triangle_{x} u+2 \varepsilon^{\beta-1} \operatorname{div}_{y} \nabla_{x} u-\nabla b+\varepsilon^{\beta-1} \nabla_{x} p
\end{aligned}
$$

Remark 5.2 Due to the parabolic regularization of the Stokes equation (16) in the variable $y$, the trace of the terms $\partial_{\tau}^{k} \partial_{x}^{\alpha} \partial_{y}^{\beta}(p, u)\left(\frac{t}{\varepsilon^{2-\beta}}, x, \frac{x}{\varepsilon}\right)$ are well defined for $k=0,1$ and $|\alpha|,|\beta| \leq 2$.

The energy equality associated to system (45) reads

$$
\begin{aligned}
& \left\|\alpha^{\varepsilon}(t)\right\|_{L^{2}\left(\mathbb{R}_{\varepsilon}^{d}\right)}^{2}+\left\|\beta^{\varepsilon}(t)\right\|_{L^{2}\left(\mathbb{R}_{\varepsilon}^{d}\right)}^{2}+2 \varepsilon^{\beta} \int_{0}^{t}\left\|\nabla \beta^{\varepsilon}(s)\right\|_{L^{2}\left(\mathbb{R}_{\varepsilon}^{d}\right)}^{2}= \\
& \left\|\alpha^{\varepsilon}(0)\right\|_{L^{2}\left(\mathbb{R}_{\varepsilon}^{d}\right)}^{2}+\left\|\beta^{\varepsilon}(0)\right\|_{L^{2}\left(\mathbb{R}_{\varepsilon}^{d}\right)}^{2}+2 \int_{0}^{t} \int_{\mathbb{R}_{\varepsilon}^{d}}\left(F^{\varepsilon} \alpha^{\varepsilon}+G^{\varepsilon} \cdot \beta^{\varepsilon}\right),
\end{aligned}
$$

and we now estimate the term

$$
\int_{0}^{t} \int_{\mathbb{R}_{\varepsilon}^{d}}\left(F^{\varepsilon} \alpha^{\varepsilon}+G^{\varepsilon} \cdot \beta^{\varepsilon}\right) .
$$


We write

$$
\int_{\mathbb{R}_{\varepsilon}^{d}} F^{\varepsilon} \alpha^{\varepsilon}=F_{1}+F_{2}
$$

and we obtain the following bounds

$$
\begin{aligned}
\left|F_{1}\right| \leq & \varepsilon^{4 \beta-6} \int_{0}^{t}\left\|\partial_{\tau} p\left(\frac{s}{\varepsilon^{2-\beta}}, x, \frac{x}{\varepsilon}\right)\right\|_{L^{2}\left(\mathbb{R}_{\varepsilon}^{d}\right)}^{2} d s+\int_{0}^{t}\left\|\alpha^{\varepsilon}\right\|_{L^{2}\left(\mathbb{R}_{\varepsilon}^{d}\right)}^{2} \\
& \leq \varepsilon^{3 \beta-4} \int_{0}^{\infty}\left\|\partial_{\tau} p\left(\tau, x, \frac{x}{\varepsilon}\right)\right\|_{L^{2}\left(\mathbb{R}_{\varepsilon}^{d}\right)}^{2} d \tau+\int_{0}^{t}\left\|\alpha^{\varepsilon}\right\|_{L^{2}\left(\mathbb{R}_{\varepsilon}^{d}\right)}^{2} \\
& \leq \varepsilon^{3 \beta-4} \int_{0}^{\infty}\left\|\partial_{\tau} p(\tau, x, y)\right\|_{L^{2}\left(\mathbb{R}^{d} \times Y_{f}\right)}^{2} d \tau+\int_{0}^{t}\left\|\alpha^{\varepsilon}\right\|_{L^{2}\left(\mathbb{R}_{\varepsilon}^{d}\right)}^{2} \\
& \leq C \varepsilon^{3 \beta-4}+\int_{0}^{t}\left\|\alpha^{\varepsilon}\right\|_{L^{2}\left(\mathbb{R}_{\varepsilon}^{d}\right)}^{2}, \\
\left|F_{2}\right| \leq & C \int_{0}^{t}\left\|\operatorname{div}_{x} u\left(\frac{s}{\varepsilon^{2-\beta}}, x, \frac{x}{\varepsilon}\right)\right\|_{L^{2}\left(\mathbb{R}_{\varepsilon}^{d}\right)}^{2} d s+\int_{0}^{t}\left\|\alpha^{\varepsilon}(s)\right\|_{L^{2}\left(\mathbb{R}_{\varepsilon}^{d}\right)}^{2} \\
\leq & C \varepsilon^{2-\beta} \int_{0}^{\infty}\left\|\operatorname{div}_{x} u\left(\tau, x, \frac{x}{\varepsilon}\right)\right\|_{L^{2}\left(\mathbb{R}_{\varepsilon}^{d}\right)}^{2} d \tau+\int_{0}^{t}\left\|\alpha^{\varepsilon}(s)\right\|_{L^{2}\left(\mathbb{R}_{\varepsilon}^{d}\right)}^{2} \\
\leq & C \varepsilon^{2-\beta} \int_{0}^{\infty}\left\|\operatorname{div}_{x} u(\tau, x, y)\right\|_{L^{2}\left(\mathbb{R}^{d} \times Y_{f}\right)}^{2} d \tau+\int_{0}^{t}\left\|\alpha^{\varepsilon}(s)\right\|_{L^{2}\left(\mathbb{R}_{\varepsilon}^{d}\right)}^{2} \\
\leq & C \varepsilon^{2-\beta}+\int_{0}^{t}\left\|\alpha^{\varepsilon}(s)\right\|_{L^{2}\left(\mathbb{R}_{\varepsilon}^{d}\right)}^{2} .
\end{aligned}
$$

We now explain the estimates above. The first inequality in the estimates of $F_{1}, F_{2}$ is just an application of the Cauchy-Schwartz inequality. The second inequality is the change of variable $s=\varepsilon^{2-\beta} \tau$. The third inequality in the estimates follows from strong two-scale convergence and hence holds for $\varepsilon$ small enough.

We now justify the energy bounds on $\partial_{\tau} p(\tau, x, y)$ and $\operatorname{div}_{x} u(\tau, x, y)$, beginning with $\operatorname{div}_{x} u$. First, notice that $\partial_{x}^{\alpha} u$ satisfies the same system (44) as $u$ with the initial data $\partial_{x}^{\alpha} u(t=0)=\partial_{x}^{\alpha} P_{y} a_{0}$. Furthermore, we have the following energy equality

$$
\left\|\partial_{x}^{\alpha} u(\tau)\right\|_{L^{2}\left(\mathbb{R}^{d} \times Y_{f}\right)}^{2}+2 \int_{0}^{\tau}\left\|\partial_{x}^{\alpha} \nabla_{y} u\left(\tau^{\prime}\right)\right\|_{L^{2}\left(\mathbb{R}^{d} \times Y_{f}\right)}^{2}=\left\|\partial_{x}^{\alpha} P_{y} a_{0}\right\|_{L^{2}\left(\mathbb{R}^{d} \times Y_{f}\right)}^{2} .
$$

By the Poincaré inequality on the cell $Y_{f}$, we also deduce that

$$
\int_{0}^{\tau}\left\|\partial_{x}^{\alpha} u\left(\tau^{\prime}\right)\right\|_{L^{2}\left(\mathbb{R}^{d} \times Y_{f}\right)}^{2} \leq C \int_{0}^{\tau}\left\|\partial_{x}^{\alpha} \nabla_{y} u\left(\tau^{\prime}\right)\right\|_{L^{2}\left(\mathbb{R}^{d} \times Y_{f}\right)}^{2} .
$$

Hence,

$$
\int_{0}^{\infty}\left\|\operatorname{div}_{x} u(\tau, x, y)\right\|_{L^{2}\left(\mathbb{R}^{d} \times Y_{f}\right)}^{2} \leq C .
$$

To obtain the energy bound for $\partial_{\tau} p(t, x, y)$, we begin by taking a time derivative of the vector equation in (44)

$$
\partial_{\tau \tau} u-\triangle_{y} \partial_{\tau} u+\nabla_{y} \partial_{\tau} p=0 .
$$

We impose the following compatibility conditions on the initial data $\partial_{\tau} u(0)$ :

$$
\partial_{\tau} u(0)=0=\nabla_{y} \partial_{\tau} u(0) \text { on } \partial Y_{s} .
$$


Multiplying (51) by $\partial_{\tau} u$ and $\partial_{\tau \tau} u$ and integrating by parts over $Y_{f}$ yields the equalities

$$
\begin{gathered}
\left\|\partial_{\tau} u(\tau)\right\|_{L^{2}\left(\mathbb{R}^{d} \times Y_{f}\right)}^{2}+2 \int_{0}^{\tau}\left\|\nabla \partial_{\tau^{\prime}} u\left(\tau^{\prime}\right)\right\|_{L^{2}\left(\mathbb{R}^{d} \times Y_{f}\right)}^{2}=\left\|\partial_{\tau} u(0)\right\|_{L^{2}\left(\mathbb{R}^{d} \times Y_{f}\right)}^{2}, \\
2 \int_{0}^{\tau}\left\|\partial_{\tau^{\prime} \tau^{\prime}} u\left(\tau^{\prime}\right)\right\|_{L^{2}\left(\mathbb{R} \times Y_{f}\right)}^{2}+\left\|\nabla_{y} \partial_{\tau} u(\tau)\right\|_{L^{2}\left(\mathbb{R} \times Y_{f}\right)}^{2}=\left\|\nabla_{y} \partial_{\tau} u(0)\right\|_{L^{2}\left(\mathbb{R} \times Y_{f}\right)}^{2} .
\end{gathered}
$$

Now using equation (51), along with the energy bounds (49), we obtain the following estimate

$$
\begin{aligned}
\int_{0}^{\tau}\left\|\partial_{\tau} p\left(\tau^{\prime}\right)\right\|_{L^{2}\left(\mathbb{R}^{d} \times Y_{f}\right)}^{2} & \leq C \int_{0}^{\tau}\left\|\nabla_{y} \partial_{\tau^{\prime}} p\left(\tau^{\prime}\right)\right\|_{L^{2}\left(\mathbb{R}^{d} ; H_{\#}^{-1}\left(Y_{f}\right)\right)}^{2} \\
& \leq C\left\|\partial_{\tau} u(0)\right\|_{L^{2}\left(\mathbb{R}_{\varepsilon}^{d}\right)}^{2} \cdot+\left\|\nabla_{y} \partial_{\tau} u(0)\right\|_{L^{2}\left(\mathbb{R} \times Y_{f}\right)}^{2} .
\end{aligned}
$$

Similarly, we write

$$
\int_{0}^{t} \int_{\mathbb{R}_{\varepsilon}^{d}} G^{\varepsilon} \cdot \beta^{\varepsilon}=G_{1}+G_{2}+G_{3}+G_{4}
$$

and we have the following estimates

$$
\begin{aligned}
& \left|G_{1}\right| \leq C \varepsilon^{\beta}\left(\int_{0}^{t}\left\|\triangle_{x} u\left(\frac{s}{\varepsilon^{2-\beta}}, x, \frac{x}{\varepsilon}\right)\right\|_{L^{2}\left(\mathbb{R}_{\varepsilon}^{d}\right)}^{2}\right)^{1 / 2}\left(\int_{0}^{t}\left\|\beta^{\varepsilon}(s)\right\|_{L^{2}\left(\mathbb{R}_{\varepsilon}^{d}\right)}^{2}\right)^{1 / 2} \\
& \leq C \varepsilon^{\beta+1}\left(\int_{0}^{t}\left\|\triangle_{x} u\left(\frac{s}{\varepsilon^{2-\beta}}, x, \frac{x}{\varepsilon}\right)\right\|_{L^{2}\left(\mathbb{R}_{\varepsilon}^{d}\right)}^{2}\right)^{1 / 2}\left(\int_{0}^{t}\left\|\nabla \beta^{\varepsilon}(s)\right\|_{L^{2}\left(\mathbb{R}_{\varepsilon}^{d}\right)}^{2}\right)^{1 / 2} \\
& \leq C \varepsilon^{2+\beta} \int_{0}^{t}\left\|\triangle_{x} u\left(\frac{s}{\varepsilon^{2-\beta}}, x, \frac{x}{\varepsilon}\right)\right\|_{L^{2}\left(\mathbb{R}_{\varepsilon}^{d}\right)}^{2}+\frac{\varepsilon^{\beta}}{5} \int_{0}^{t}\left\|\nabla \beta^{\varepsilon}(s)\right\|_{L^{2}\left(\mathbb{R}_{\varepsilon}^{d}\right)}^{2} \\
& \leq C \varepsilon^{4}+\frac{\varepsilon^{\beta}}{5} \int_{0}^{t}\left\|\nabla \beta^{\varepsilon}(s)\right\|_{L^{2}\left(\mathbb{R}_{\varepsilon}^{d}\right)}^{2}, \\
& \left|G_{2}\right| \leq C \varepsilon^{\beta-1}\left(\int_{0}^{t}\left\|\operatorname{div}_{y} \nabla_{x} u\left(\frac{s}{\varepsilon^{2-\beta}}, x, \frac{x}{\varepsilon}\right)\right\|_{L^{2}\left(\mathbb{R}_{\varepsilon}^{d}\right)}^{2}\right)^{1 / 2}\left(\int_{0}^{t}\left\|\beta^{\varepsilon}(s)\right\|_{L^{2}\left(\mathbb{R}_{\varepsilon}^{d}\right)}\right)^{1 / 2} \\
& \leq C \varepsilon^{\beta}\left(\int_{0}^{t}\left\|\operatorname{div}_{y} \nabla_{x} u\left(\frac{s}{\varepsilon^{2-\beta}}, x, \frac{x}{\varepsilon}\right)\right\|_{L^{2}\left(\mathbb{R}_{\varepsilon}^{d}\right)}^{2}\right)^{1 / 2}\left(\int_{0}^{t}\left\|\nabla \beta^{\varepsilon}(s)\right\|_{L^{2}\left(\mathbb{R}_{\varepsilon}^{d}\right)}\right)^{1 / 2} \\
& \leq C \varepsilon^{\beta} \int_{0}^{t}\left\|\operatorname{div}_{y} \nabla_{x} u\left(\frac{s}{\varepsilon^{2-\beta}}, x, \frac{x}{\varepsilon}\right)\right\|_{L^{2}\left(\mathbb{R}_{\varepsilon}^{d}\right)}^{2}+\frac{\varepsilon^{\beta}}{5} \int_{0}^{t}\left\|\nabla \beta^{\varepsilon}(s)\right\|_{L^{2}\left(\mathbb{R}_{\varepsilon}^{d}\right)}^{2} \\
& \leq C \varepsilon^{2}+\frac{\varepsilon^{\beta}}{5} \int_{0}^{t}\left\|\nabla \beta^{\varepsilon}(s)\right\|_{L^{2}\left(\mathbb{R}_{\varepsilon}^{d}\right)}^{2}, \\
& \left|G_{3}\right| \leq C\left(\int_{0}^{t}\|\nabla b\|_{L^{2}\left(\mathbb{R}_{\varepsilon}^{d}\right)}^{2}\right)^{1 / 2}\left(\int_{0}^{t}\left\|\beta^{\varepsilon}(s)\right\|_{L^{2}\left(\mathbb{R}_{\varepsilon}^{d}\right)}^{2}\right)^{1 / 2} \\
& \leq C \varepsilon\left(\int_{0}^{t}\|\nabla b\|_{L^{2}\left(\mathbb{R}_{\varepsilon}^{d}\right)}^{2}\right)^{1 / 2}\left(\int_{0}^{t}\left\|\nabla \beta^{\varepsilon}(s)\right\|_{L^{2}\left(\mathbb{R}_{\varepsilon}^{d}\right)}^{2}\right)^{1 / 2} \\
& \leq C \varepsilon^{2-\beta} \int_{0}^{t}\|\nabla b\|_{L^{2}\left(\mathbb{R}_{\varepsilon}^{d}\right)}^{2}+\frac{\varepsilon^{\beta}}{5} \int_{0}^{t}\left\|\nabla \beta^{\varepsilon}(s)\right\|_{2}^{2} \\
& \leq C t \varepsilon^{2-\beta}+\frac{\varepsilon^{\beta}}{5} \int_{0}^{t}\left\|\nabla \beta^{\varepsilon}(s)\right\|_{2}^{2},
\end{aligned}
$$




$$
\begin{aligned}
\left|G_{4}\right| & \leq C \varepsilon^{\beta-1}\left(\int_{0}^{t}\left\|\nabla_{x} p\left(\frac{s}{\varepsilon^{2-\beta}}, x, \frac{x}{\varepsilon}\right)\right\|_{L^{2}\left(\mathbb{R}_{\varepsilon}^{d}\right)}^{2}\right)^{1 / 2}\left(\int_{0}^{t}\left\|\beta^{\varepsilon}(s)\right\|_{L^{2}\left(\mathbb{R}_{\varepsilon}^{d}\right)}^{2}\right)^{1 / 2} \\
& \leq C \varepsilon^{\beta}\left(\int_{0}^{t}\left\|\nabla_{x} p\left(\frac{s}{\varepsilon^{2-\beta}}, x, \frac{x}{\varepsilon}\right)\right\|_{L^{2}\left(\mathbb{R}_{\varepsilon}^{d}\right)}^{2}\right)^{1 / 2}\left(\int_{0}^{t}\left\|\nabla \beta^{\varepsilon}(s)\right\|_{L^{2}\left(\mathbb{R}_{\varepsilon}^{d}\right)}^{2}\right)^{1 / 2} \\
& \leq C \varepsilon^{2} \int_{0}^{\infty}\left\|\nabla_{x} p\left(\tau, x, \frac{x}{\varepsilon}\right)\right\|_{L^{2}\left(\mathbb{R}_{\varepsilon}^{d}\right)}^{2}+\frac{\varepsilon^{\beta}}{5} \int_{0}^{t}\left\|\nabla \beta^{\varepsilon}(s)\right\|_{L^{2}\left(\mathbb{R}_{\varepsilon}^{d}\right)}^{2} \\
& \leq C \varepsilon^{2}+\frac{\varepsilon^{\beta}}{5} \int_{0}^{t}\left\|\nabla \beta^{\varepsilon}(s)\right\|_{L^{2}\left(\mathbb{R}_{\varepsilon}^{d}\right)}^{2} .
\end{aligned}
$$

Here, the second inequality in the estimates of $G_{1}, G_{2}$ made use of the Poincaré inequality on the domain $\mathbb{R}_{\varepsilon}^{d}$. We concluded the final inequality in the estimates $G_{1}, G_{2}$ with a change of variable in time along with the bounds in (49), (50).

Combining all the estimates on the force terms, the energy bound (46) becomes

$$
\begin{aligned}
& \left\|\alpha^{\varepsilon}(t)\right\|_{L^{2}\left(\mathbb{R}_{\varepsilon}^{d}\right)}^{2}+\left\|\beta^{\varepsilon}(t)\right\|_{L^{2}\left(\mathbb{R}_{\varepsilon}^{d}\right)}^{2}+\frac{\varepsilon^{\beta}}{5} \int_{0}^{t}\left\|\nabla \beta^{\varepsilon}(s)\right\|_{L^{2}\left(\mathbb{R}_{\varepsilon}^{d}\right)}^{2} \\
& \leq\left\|\alpha^{\varepsilon}(0)\right\|_{L^{2}\left(\mathbb{R}_{\varepsilon}^{d}\right)}^{2}+\left\|\beta^{\varepsilon}(0)\right\|_{L^{2}\left(\mathbb{R}_{\varepsilon}^{d}\right)}^{2}+\int_{0}^{t}\left\|\alpha^{\varepsilon}(s)\right\|_{L^{2}\left(\mathbb{R}_{\varepsilon}^{d}\right)}^{2}+C_{t, \varepsilon}
\end{aligned}
$$

where

$$
C_{t, \varepsilon}=C\left(\varepsilon^{3 \beta-4}+\varepsilon^{2-\beta}+\varepsilon^{4}+\varepsilon^{2}+t \varepsilon^{2-\beta}\right) .
$$

Hence, by applying Gronwall's lemma to (53), we conclude the proof of Theorem 3.6 for $\beta \in(4 / 3,2)$

To extend this result to $\beta \in(1,4 / 3]$, we take $l>0$ large enough such that $(4 l+3)(\beta-1)-1>0$ and proceed with a similar analysis as above. Notice that we have

$$
\varepsilon^{(4 l+4)(\beta-1)-2} \int_{0}^{t} \int_{\mathbb{R}_{\varepsilon}^{d}}\left|\partial_{\tau} p_{l}\right|^{2}=O\left(\varepsilon^{m_{l}(\beta-1)-1}\right)
$$

where $m_{l}=4 l+3$, hence the proof of theorem 3.6 is now complete.

\section{$5.2(\beta=2)$ Proof of theorem 3.8:}

We define the following error terms

$$
\begin{aligned}
& \alpha^{\varepsilon}=p^{\varepsilon}-p(t, x)-\varepsilon p_{1}\left(t, x, \frac{x}{\varepsilon}\right)-\varepsilon p_{2}(t, x)-\varepsilon^{2} p_{3}\left(t, x, \frac{x}{\varepsilon}\right)-q^{\varepsilon}, \\
& \beta^{\varepsilon}=u^{\varepsilon}-u\left(t, x, \frac{x}{\varepsilon}\right)-\varepsilon u_{1}\left(t, x, \frac{x}{\varepsilon}\right)-v^{\varepsilon}
\end{aligned}
$$

where $\left(p_{2}(t, x), p_{3}(t, x, y), u_{1}(t, x, y)\right)$ uniquely satisfies the two-scale system

$$
\left\{\begin{array}{cc}
\left|Y_{f}\right| \partial_{t} p_{2}+\operatorname{div}_{x} \int_{Y_{f}} u_{1}=0 & \text { in } \mathbb{R}^{d} \\
\partial_{t} u_{1}-\triangle_{y} u_{1}+\nabla_{x} p_{2}+\nabla_{y} p_{3}=-\nabla_{x} p_{1} & \text { in } \mathbb{R}^{d} \times Y_{f} \\
\operatorname{div}_{y} u_{1}=\operatorname{div}_{x} u-\operatorname{div}_{x} \int_{Y_{f}} u & \\
u_{1}=0 & \text { on } \partial Y_{s} \\
\int_{Y_{f}} p_{3}=0 & \\
p(t=0)=0 & \\
u(t=0)=0 & \\
y \rightarrow\left(p_{3}, u_{1}\right) \text { is } Y \text {-periodic } . &
\end{array}\right.
$$


The system associated to the error term $\left(\alpha^{\varepsilon}, \beta^{\varepsilon}\right)$ reads

$$
\left\{\begin{aligned}
\partial_{t} \alpha^{\varepsilon}+\operatorname{div} \beta^{\varepsilon} & =F^{\varepsilon}(t,, x) & & \text { in }(0, \infty) \times \mathbb{R}_{\varepsilon}^{d} \\
\partial_{t} \beta^{\varepsilon}-\varepsilon^{\beta} \triangle \beta^{\varepsilon}+\nabla \alpha^{\varepsilon} & =G^{\varepsilon}(t, x) & & \\
\beta^{\varepsilon} & =0 & & \text { on } \partial \mathbb{R}_{\varepsilon}^{d} \\
\alpha^{\varepsilon}(t=0) & =b_{1}^{\varepsilon}-b(x)-\varepsilon p_{1}(t=0)-\varepsilon^{2} p_{3}(t=0) & & \\
\beta^{\varepsilon}(t=0) & =P_{M}^{\varepsilon} a^{\varepsilon}(x)-P_{y} a_{0}\left(x, \frac{x}{\varepsilon}\right) & &
\end{aligned}\right.
$$

where the force terms here are given by

$$
\begin{aligned}
& F^{\varepsilon}(t, x)=-\varepsilon \partial_{t} p_{1}-\varepsilon^{2} \partial_{t} p_{3} \\
& G^{\varepsilon}(t, x)=\varepsilon^{2} \triangle_{x} u+2 \varepsilon \operatorname{div}_{y} \nabla_{x} u+\varepsilon^{3} \triangle_{x} u_{1}+2 \varepsilon^{2} \operatorname{div}_{y} \nabla_{x} u_{1}-\varepsilon^{2} \nabla_{x} p_{3} .
\end{aligned}
$$

We now imitate the proof given for the case $\beta \in(1,2)$. Notice that $\left(\alpha^{\varepsilon}, \beta^{\varepsilon}\right)$ satisfies the energy bound in (46) (for $\beta=2$ ), hence it suffices to show

$$
\begin{gathered}
\left|\int_{0}^{t} \int_{\mathbb{R}_{\varepsilon}^{d}} F^{\varepsilon} \alpha^{\varepsilon}+G^{\varepsilon} \cdot \beta^{\varepsilon}\right| \\
\leq O\left(\varepsilon^{\gamma}\right)+C_{1} \varepsilon^{2} \int_{0}^{t}\left\|\nabla \beta^{\varepsilon}(s)\right\|_{L^{2}\left(\mathbb{R}_{\varepsilon}^{d}\right)}^{2}+C \int_{0}^{t}\left\|\alpha^{\varepsilon}(s)\right\|_{L^{2}\left(\mathbb{R}_{\varepsilon}^{d}\right)}^{2},
\end{gathered}
$$

for some $0<\gamma$ and constant $C_{1}<1$.

We begin by writing

$$
\int_{\mathbb{R}_{\varepsilon}^{d}} F^{\varepsilon} \alpha^{\varepsilon}=F_{1}+F_{2}
$$

and we obtain the following bounds

$$
\begin{aligned}
\left|F_{1}\right| & \leq \varepsilon^{2} \int_{0}^{\infty}\left\|\partial_{\tau} p_{1}\left(t, x, \frac{x}{\varepsilon}\right)\right\|_{L^{2}\left(\mathbb{R}_{\varepsilon}^{d}\right)}^{2}+\int_{0}^{t}\left\|\alpha^{\varepsilon}\right\|_{L^{2}\left(\mathbb{R}_{\varepsilon}^{d}\right)}^{2} \\
& \leq \varepsilon^{2} \int_{0}^{\infty}\left\|\partial_{\tau} p_{1}(t, x, y)\right\|_{L^{2}\left(\mathbb{R}^{d} \times Y_{f}\right)}^{2}+\int_{0}^{t}\left\|\alpha^{\varepsilon}\right\|_{L^{2}\left(\mathbb{R}_{\varepsilon}^{d}\right)}^{2} \\
& \leq C \varepsilon^{2}+\int_{0}^{t}\left\|\alpha^{\varepsilon}\right\|_{L^{2}\left(\mathbb{R}_{\varepsilon}^{d}\right)}^{2}, \\
\left|F_{2}\right| & \leq \varepsilon^{4} \int_{0}^{\infty}\left\|\partial_{\tau} p_{3}\left(t, x, \frac{x}{\varepsilon}\right)\right\|_{L^{2}\left(\mathbb{R}_{\varepsilon}^{d}\right)}^{2}+\int_{0}^{t}\left\|\alpha^{\varepsilon}\right\|_{L^{2}\left(\mathbb{R}_{\varepsilon}^{d}\right)}^{2} \\
& \leq \varepsilon^{4} \int_{0}^{\infty}\left\|\partial_{\tau} p_{3}(t, x, y)\right\|_{L^{2}\left(\mathbb{R}^{d} \times Y_{f}\right)}^{2}+\int_{0}^{t}\left\|\alpha^{\varepsilon}\right\|_{L^{2}\left(\mathbb{R}_{\varepsilon}^{d}\right)}^{2} \\
& \leq C \varepsilon^{4}+\int_{0}^{t}\left\|\alpha^{\varepsilon}\right\|_{L^{2}\left(\mathbb{R}_{\varepsilon}^{d}\right)}^{2} .
\end{aligned}
$$

We now justify the energy bound on $\partial_{t} p_{1}(\tau, x, y)$ (the energy bound for $\partial_{t} p_{3}$ is obtained similarly). Notice, it suffices to show that $\nabla_{y} \partial_{t} p_{1}$ is bounded in $L^{2}\left((0, \infty) \times \mathbb{R}^{d} ; H_{\#}^{-1}\left(Y_{f}\right)\right)^{d}$. We begin by taking a time derivative of the vector equation in (17)

$$
\partial_{t t} u-\triangle_{y} \partial_{t} u+\nabla_{x} \partial_{t} p+\nabla_{y} \partial_{t} p_{1}=0 .
$$

Multiplying (58) by $\partial_{t} u$ and $\partial_{t t} u$ and integrating by parts over $Y_{f}$ yields the equality

$$
\begin{aligned}
& \left\|\partial_{t} u(t)\right\|_{L^{2}\left(\mathbb{R}^{d} \times Y_{f}\right)}^{2}+\left|Y_{f}\right|\left\|\partial_{t} p(t)\right\|_{L^{2}\left(\mathbb{R}^{d}\right)}^{2}+2 \int_{0}^{t}\left\|\nabla_{y} \partial_{s} u(s)\right\|_{L^{2}\left(\mathbb{R}^{d} \times Y_{f}\right)}^{2} \\
& =\left\|\partial_{t} u(0)\right\|_{L^{2}\left(\mathbb{R}^{d} \times Y_{f}\right)}^{2}+\left|Y_{f}\right|\left\|\partial_{t} p(0)\right\|_{L^{2}\left(\mathbb{R}^{d}\right)}^{2},
\end{aligned}
$$


and the inequality

$$
\begin{aligned}
& \int_{0}^{t}\left\|\partial_{s s} u(s)\right\|_{L^{2}\left(\mathbb{R}^{d} \times Y_{f}\right)}^{2}+\frac{1}{2}\left\|\nabla_{y} \partial_{t} u(t)\right\|_{L^{2}\left(\mathbb{R}^{d} \times Y_{f}\right)}^{2} \\
& =\frac{1}{2}\left\|\nabla_{y} \partial_{t} u(0)\right\|_{L^{2}\left(\mathbb{R}^{d} \times Y_{f}\right)}^{2}-\int_{0}^{t} \int_{\mathbb{R}^{d} \times Y_{f}} \nabla_{x} \partial_{t} p \cdot \partial_{t t} u \\
& \leq \frac{1}{2}\left\|\nabla_{y} \partial_{t} u(0)\right\|_{L^{2}\left(\mathbb{R}^{d} \times Y_{f}\right)}^{2}+\frac{\left|Y_{f}\right|}{2} \int_{0}^{t}\left\|\nabla_{x} \partial_{s} p(s)\right\|_{L^{2}\left(\mathbb{R}^{d} \times Y_{f}\right)}^{2}+\frac{1}{2} \int_{0}^{t}\left\|\partial_{s s} u(s)\right\|_{L^{2}\left(\mathbb{R}^{d} \times Y_{f}\right)}^{2} .
\end{aligned}
$$

Next, we observe that $\partial_{x}^{\alpha}\left(p, p_{1}, u\right)$ satisfies the same system $(17)$ as $\left(p, p_{1}, u\right)$ with the initial data $\partial_{x}^{\alpha}\left(b, P_{y} a_{0}\right)$. Moreover, we have the following energy equality

$$
\begin{aligned}
& \left\|\partial_{x}^{\alpha} u(t)\right\|_{L^{2}\left(\mathbb{R}^{d} \times Y_{f}\right)}^{2}+\left|Y_{f}\right|\left\|\partial_{x}^{\alpha} p(t)\right\|_{L^{2}\left(\mathbb{R}^{d}\right)}^{2}+2 \int_{0}^{t}\left\|\nabla_{y} \partial_{x}^{\alpha} u(s)\right\|_{L^{2}\left(\mathbb{R}^{d} \times Y_{f}\right)}^{2} \\
& =\left|Y_{f}\right|\left\|\partial_{x}^{\alpha} b\right\|_{L^{2}\left(\mathbb{R}^{d} \times Y_{f}\right)}^{2}+\left\|\partial_{x}^{\alpha} P_{y} a_{0}\right\|_{L^{2}\left(\mathbb{R}^{d}\right)}^{2}
\end{aligned}
$$

Taking a time derivative of the scalar equation in (17) yields

$$
\left|Y_{f}\right| \partial_{x}^{\alpha} \partial_{t} p+\partial_{x}^{\alpha} \operatorname{div} x \int_{Y_{f}} u=0
$$

and we deduce the inequality

$$
\begin{aligned}
\int_{0}^{t}\left\|\partial_{x}^{\alpha} \partial_{s} p(s)\right\|_{L^{2}\left(\mathbb{R}^{d}\right)}^{2} & \leq C \int_{0}^{t}\left\|\partial_{x}^{\alpha} \operatorname{div}_{x} u(t)\right\|_{L^{2}\left(\mathbb{R}^{d} \times Y_{f}\right)}^{2} \\
& \leq C \int_{0}^{t}\left\|\partial_{x}^{\alpha} \operatorname{div}_{x} \nabla_{y} u(t)\right\|_{L^{2}\left(\mathbb{R}^{d} \times Y_{f}\right)}^{2}
\end{aligned}
$$

Here, the last inequality in (62) follows from the Poincaré inequality in the cell $Y_{f}$. Combing the estimates (59), (60), (61), (62) we conclude

$$
\left\|\nabla_{y} \partial_{t} p_{1}\right\|_{L^{2}\left((0, \infty) \times \mathbb{R}^{d} ; H_{\#}^{-1}\left(Y_{f}\right)\right)} \leq C
$$

Similarly, we write

$$
\int_{0}^{t} \int_{\mathbb{R}_{\varepsilon}^{d}} G^{\varepsilon} \cdot \beta^{\varepsilon}=G_{1}+G_{2}+G_{3}+G_{4}+G_{5}
$$

and we have the following estimates 


$$
\begin{aligned}
& \left|G_{1}\right| \leq C \varepsilon^{2}\left(\int_{0}^{\infty}\left\|\triangle_{x} u\left(t, x, \frac{x}{\varepsilon}\right)\right\|_{L^{2}\left(\mathbb{R}_{\varepsilon}^{d}\right)}^{2}\right)^{1 / 2}\left(\int_{0}^{t}\left\|\beta^{\varepsilon}(s)\right\|_{L^{2}\left(\mathbb{R}_{\varepsilon}^{d}\right)}^{2}\right)^{1 / 2} \\
& \leq C \varepsilon^{3}\left(\int_{0}^{\infty}\left\|\triangle_{x} u\left(t, x, \frac{x}{\varepsilon}\right)\right\|_{L^{2}\left(\mathbb{R}_{\varepsilon}^{d}\right)}^{2}\right)^{1 / 2}\left(\int_{0}^{t}\left\|\nabla \beta^{\varepsilon}(s)\right\|_{L^{2}\left(\mathbb{R}_{\varepsilon}^{d}\right)}^{2}\right)^{1 / 2} \\
& \leq C \varepsilon^{4} \int_{0}^{\infty}\left\|\triangle_{x} u\left(t, x, \frac{x}{\varepsilon}\right)\right\|_{L^{2}\left(\mathbb{R}_{\varepsilon}^{d}\right)}^{2}+\frac{\varepsilon^{2}}{6} \int_{0}^{t}\left\|\nabla \beta^{\varepsilon}(s)\right\|_{L^{2}\left(\mathbb{R}_{\varepsilon}^{d}\right)}^{2} \\
& \leq C \varepsilon^{4}+\frac{\varepsilon^{2}}{6} \int_{0}^{t}\left\|\nabla \beta^{\varepsilon}(s)\right\|_{L^{2}\left(\mathbb{R}_{\varepsilon}^{d}\right)}^{2}, \\
& \left|G_{2}\right| \leq C \varepsilon\left(\int_{0}^{\infty}\left\|\operatorname{div}_{y} \nabla_{x} u\left(t, x, \frac{x}{\varepsilon}\right)\right\|_{L^{2}\left(\mathbb{R}_{\varepsilon}^{d}\right)}^{2}\right)^{1 / 2}\left(\int_{0}^{t}\left\|\beta^{\varepsilon}(s)\right\|_{L^{2}\left(\mathbb{R}_{\varepsilon}^{d}\right)}\right)^{1 / 2} \\
& \leq C \varepsilon^{2}\left(\int_{0}^{\infty}\left\|\operatorname{div}_{y} \nabla_{x} u\left(t, x, \frac{x}{\varepsilon}\right)\right\|_{L^{2}\left(\mathbb{R}_{\varepsilon}^{d}\right)}^{2}\right)^{1 / 2}\left(\int_{0}^{t}\left\|\nabla \beta^{\varepsilon}(s)\right\|_{L^{2}\left(\mathbb{R}_{\varepsilon}^{d}\right)}\right)^{1 / 2} \\
& \leq C \varepsilon^{2} \int_{0}^{\infty}\left\|\operatorname{div}_{y} \nabla_{x} u\left(t, x, \frac{x}{\varepsilon}\right)\right\|_{L^{2}\left(\mathbb{R}_{\varepsilon}^{d}\right)}^{2}+\frac{\varepsilon^{2}}{6} \int_{0}^{t}\left\|\nabla \beta^{\varepsilon}(s)\right\|_{L^{2}\left(\mathbb{R}_{\varepsilon}^{d}\right)}^{2} \\
& \leq C \varepsilon^{2}+\frac{\varepsilon^{2}}{6} \int_{0}^{t}\left\|\nabla \beta^{\varepsilon}(s)\right\|_{L^{2}\left(\mathbb{R}_{\varepsilon}^{d}\right)}^{2}, \\
& \left|G_{3}\right| \leq C \varepsilon^{3}\left(\int_{0}^{\infty}\left\|\triangle_{x} u_{1}\left(t, x, \frac{x}{\varepsilon}\right)\right\|_{L^{2}\left(\mathbb{R}_{\varepsilon}^{d}\right)}^{2}\right)^{1 / 2}\left(\int_{0}^{t}\left\|\beta^{\varepsilon}(s)\right\|_{L^{2}\left(\mathbb{R}_{\varepsilon}^{d}\right)}^{2}\right)^{1 / 2} \\
& \leq C \varepsilon^{4}\left(\int_{0}^{\infty}\left\|\triangle_{x} u_{1}\left(t, x, \frac{x}{\varepsilon}\right)\right\|_{L^{2}\left(\mathbb{R}_{\varepsilon}^{d}\right)}^{2}\right)^{1 / 2}\left(\int_{0}^{t}\left\|\nabla \beta^{\varepsilon}(s)\right\|_{L^{2}\left(\mathbb{R}_{\varepsilon}^{d}\right)}^{2}\right)^{1 / 2} \\
& \leq C \varepsilon^{6} \int_{0}^{\infty}\left\|\triangle_{x} u_{1}\left(t, x, \frac{x}{\varepsilon}\right)\right\|_{L^{2}\left(\mathbb{R}_{\varepsilon}^{d}\right)}^{2}+\frac{\varepsilon^{2}}{6} \int_{0}^{t}\left\|\nabla \beta^{\varepsilon}(s)\right\|_{L^{2}\left(\mathbb{R}_{\varepsilon}^{d}\right)}^{2} \\
& \leq C \varepsilon^{6}+\frac{\varepsilon^{2}}{6} \int_{0}^{t}\left\|\nabla \beta^{\varepsilon}(s)\right\|_{L^{2}\left(\mathbb{R}_{\varepsilon}^{d}\right)}^{2}, \\
& \left|G_{4}\right| \leq C \varepsilon^{2}\left(\int_{0}^{\infty}\left\|\operatorname{div}_{y} \nabla_{x} u_{1}\left(t, x, \frac{x}{\varepsilon}\right)\right\|_{L^{2}\left(\mathbb{R}_{\varepsilon}^{d}\right)}^{2}\right)^{1 / 2}\left(\int_{0}^{t}\left\|\beta^{\varepsilon}(s)\right\|_{L^{2}\left(\mathbb{R}_{\varepsilon}^{d}\right)}\right)^{1 / 2} \\
& \leq C \varepsilon^{3}\left(\int_{0}^{\infty}\left\|\operatorname{div}_{y} \nabla_{x} u_{1}\left(t, x, \frac{x}{\varepsilon}\right)\right\|_{L^{2}\left(\mathbb{R}_{\varepsilon}^{d}\right)}^{2}\right)^{1 / 2}\left(\int_{0}^{t}\left\|\nabla \beta^{\varepsilon}(s)\right\|_{L^{2}\left(\mathbb{R}_{\varepsilon}^{d}\right)}\right)^{1 / 2} \\
& \leq C \varepsilon^{4} \int_{0}^{\infty}\left\|\operatorname{div}_{y} \nabla_{x} u_{1}\left(t, x, \frac{x}{\varepsilon}\right)\right\|_{L^{2}\left(\mathbb{R}_{\varepsilon}^{d}\right)}^{2}+\frac{\varepsilon^{2}}{6} \int_{0}^{t}\left\|\nabla \beta^{\varepsilon}(s)\right\|_{L^{2}\left(\mathbb{R}_{\varepsilon}^{d}\right)}^{2}, \\
& \leq C \varepsilon^{4}+\frac{\varepsilon^{2}}{6} \int_{0}^{t}\left\|\nabla \beta^{\varepsilon}(s)\right\|_{L^{2}\left(\mathbb{R}_{\varepsilon}^{d}\right)}^{2}, \\
& \left|G_{5}\right| \leq C \varepsilon^{2}\left(\int_{0}^{t}\left\|\nabla_{x} p_{3}\left(t, x, \frac{x}{\varepsilon}\right)\right\|_{L^{2}\left(\mathbb{R}_{\varepsilon}^{d}\right)}^{2}\right)^{1 / 2}\left(\int_{0}^{t}\left\|\beta^{\varepsilon}(s)\right\|_{L^{2}\left(\mathbb{R}_{\varepsilon}^{d}\right)}^{2}\right)^{1 / 2} \\
& \leq C \varepsilon^{3}\left(\int_{0}^{t}\left\|\nabla_{x} p_{3}\left(t, x, \frac{x}{\varepsilon}\right)\right\|_{L^{2}\left(\mathbb{R}_{\varepsilon}^{d}\right)}^{2}\right)^{1 / 2}\left(\int_{0}^{t}\left\|\nabla \beta^{\varepsilon}(s)\right\|_{L^{2}\left(\mathbb{R}_{\varepsilon}^{d}\right)}^{2}\right)^{1 / 2} \\
& \leq C \varepsilon^{4} \int_{0}^{\infty}\left\|\nabla_{x} p_{3}\left(t, x, \frac{x}{\varepsilon}\right)\right\|_{L^{2}\left(\mathbb{R}_{\varepsilon}^{d}\right)}^{2}+\frac{\varepsilon^{2}}{6} \int_{0}^{t}\left\|\nabla \beta^{\varepsilon}(s)\right\|_{L^{2}\left(\mathbb{R}_{\varepsilon}^{d}\right)}^{2} \\
& \leq C \varepsilon^{4}+\frac{\varepsilon^{2}}{6} \int_{0}^{t}\left\|\nabla \beta^{\varepsilon}(s)\right\|_{L^{2}\left(\mathbb{R}_{\varepsilon}^{d}\right)}^{2} .
\end{aligned}
$$

We remark that the final bounds in $G_{1}, G_{2}$ make use of (61) and the Poincaré inequality on the cell $Y_{f}$. We do not provide the energy bounds for the terms $\triangle_{x} u_{1}, \operatorname{div}_{y} \nabla_{x} u_{1}, \nabla_{x} p_{3}$, but note that they can be obtained similarly to the methods presented here. 
Combing all the estimates associated with the scalar and vector force terms, (56) holds for $\gamma=2$ and $C_{1}=5 / 6$. The proof of Theorem 3.8 is now complete.

\section{$5.3(2<\beta)$ Proof of theorem 3.10:}

In the case $2<\beta$, there is a boundary layer in space of size $\varepsilon^{\beta / 2}$ associated to the micro-incompressible part of the flow. The asymptotic analysis necessary in the case $2<\beta$ is interesting in its own right. We will only detail the construction of the complete asymptotic analysis in the case $2<\beta$. The convergence proof based on the estimate of the error terms $\left(\alpha^{\varepsilon}, \beta^{\varepsilon}\right)$ follows exactly as in the previous two proofs and will be omitted.

We introduce the following asymptotic expansions

$$
\begin{aligned}
& p^{\varepsilon}-q^{\varepsilon} \sim \sum_{k, l} \varepsilon^{k+l(\beta-2) / 2}\left(p_{k, l}\left(t, x, \frac{x}{\varepsilon}\right)+p_{k, l}^{b d y}\left(t, x, \Pi\left(\frac{x}{\varepsilon}\right), \frac{d\left(\frac{x}{\varepsilon}\right)}{\varepsilon^{(\beta-2) / 2}}\right) \chi\left(\frac{x}{\varepsilon}\right)\right) \\
& u^{\varepsilon}-v^{\varepsilon} \sim \sum_{k, l} \varepsilon^{k+l(\beta-2) / 2}\left(u_{k, l}\left(t, x, \frac{x}{\varepsilon}\right)+u_{k, l}^{b d y}\left(t, x, \Pi\left(\frac{x}{\varepsilon}\right), \frac{d\left(\frac{x}{\varepsilon}\right)}{\varepsilon^{(\beta-2) / 2}}\right) \chi\left(\frac{x}{\varepsilon}\right)\right)
\end{aligned}
$$

Here, $\Pi y$ is the projection of $y$ on the boundary $\partial Y_{s}$ and $d(y)$ denotes the distance function to the boundary, namely the distance between $y$ and $\partial Y_{s}$. Note that $\Pi$ is well defined in a small neighborhood of the boundary $\partial Y_{s}$. The function $\chi(y) \in C_{0}^{\infty}(Y)$ is a smooth cut-off function such that $\chi(y)=1$ in a neighborhood of $Y_{s}$ and $\chi(y)=0$ if $d(y)>\delta$ for some $\delta$ small enough. Here $\delta>0$ is taken in such a way that $\Pi$ is uniquely determined in the neighborhood $\{y \mid 0<d(y)<\delta\}$ and $\nabla d$ is a unit normal vector on $\operatorname{supp}(\chi) \cap Y_{f}$. We will use $\nu=\nabla d$ to denote the inward normal vector to $Y_{f}$. An important remark to keep in mind is that we only need $\left(p_{k, l}^{b d y}, u_{k, l}^{b d y}\right)$ when $y$ is close to the boundary $\partial Y_{s}$ which justifies the presence of the cut-off function $\chi\left(\frac{x}{\varepsilon}\right)$ in $(63)$.

The boundary layer terms $\left(p_{k, l}^{b d y}, u_{k, l}^{b d y}\right)(t, x, \Pi y, \xi)$ satisfy

$$
\left(p_{k, l}^{b d y}, u_{k, l}^{b d y}\right) \rightarrow 0
$$

when $\xi$ goes to $\infty$ and

$$
u_{k, l}(t, x, y)+u_{k, l}^{b d y}(t, x, \Pi y, \xi=0)=0 \quad \text { when } \quad y=\Pi y \in \partial Y_{s}
$$

We will also need a curvilinear coordinate system $\Xi=\left(\xi^{\prime}, \xi_{d}\right), \xi^{\prime}=\left(\xi_{1}, \ldots, \xi_{d-1}\right)$ adapted to $\partial Y_{s}$ and defined in a neighborhood of $\partial Y_{s}$ such that $\partial Y_{s}=\left\{\xi_{d}=0\right\}$ and $Y_{f}$ is located at the side $\left\{\xi_{d}>0\right\}$. Of course, we think here of $\Xi \rightarrow y(\Xi)$ as a change of variables where the expansion of the operators $\Delta, \nabla$ and div will have simpler expressions. We will follow very similar notations to those in [14,13]. We define the tubular neighborhood $Y_{s, \delta}=\left\{-\delta<\xi_{d}<\delta\right\}$. We also assume that our curvilinear coordinate system $\left(\xi^{\prime}, \xi_{d}\right)$ is orthogonal and that

$$
\frac{\partial y}{\xi_{d}} \cdot \frac{\partial y}{\xi_{d}}=\sum_{1}^{d}\left(\frac{\partial y_{i}}{\xi_{d}}\right)^{2}=1
$$

which means that $\xi_{d}=d(y)=\varepsilon^{(\beta-2) / 2} \xi$ in $Y_{s, \delta} \cap Y_{f}$. We introduce the vectors

$$
g_{\alpha}=\frac{\partial y}{\xi_{\alpha}}=\left(\frac{\partial y_{1}}{\xi_{\alpha}}, \ldots, \frac{\partial y_{d}}{\xi_{\alpha}}\right), \quad 1 \leq \alpha \leq d
$$

Hence, the metric $\left(g_{\alpha \beta}\right)_{1 \leq \alpha, \beta \leq d}=\left(g_{\alpha} \cdot g_{\beta}\right)_{1 \leq \alpha, \beta \leq d}=\operatorname{diag}\left(g_{11}, \ldots, g_{d d}\right)$ and $g_{d d}=1$. We will use the notation $h_{i}=\sqrt{g_{i i}}>0, i=1, \ldots, d-1$ and $h=\sqrt{\operatorname{det}\left(\left(g_{\alpha \beta}\right)_{1 \leq \alpha, \beta \leq d}\right)}$. With these notations, we can also 
rewrite $\operatorname{div}_{y} U, \Delta_{y} f$ and $\nabla_{y} f$ in the following way. If $U$ and $f$ are defined in a neighborhood of $\partial Y_{s}$ and such that $U=\sum_{i=0}^{d} U_{i} \mathbf{e}_{i}$ where $\mathbf{e}_{i}=\frac{g_{i}}{\left|g_{i}\right|}$ and $f$ is scalar valued, then

$$
\begin{gathered}
\operatorname{div}_{y} U=\frac{1}{h} \sum_{i=1}^{d-1} \frac{\partial}{\partial \xi_{i}}\left(\frac{h}{h_{i}} U_{i}\right)+\frac{1}{h} \frac{\partial\left(h U_{d}\right)}{\partial \xi_{d}} \\
\Delta_{y} f=\frac{1}{h} \sum_{1 \leq i, j \leq d-1} \frac{\partial}{\partial \xi_{i}}\left(\frac{h}{h_{i}^{2}} \frac{\partial f}{\partial \xi_{i}}\right)+\frac{h^{\prime}}{h} \frac{\partial f}{\partial \xi_{d}}+\frac{\partial^{2} f}{\partial \xi_{d}^{2}}, \\
\nabla_{y} f=\sum_{i=1}^{d-1} \frac{1}{h_{i}} \frac{\partial f}{\partial \xi_{i}} \mathbf{e}_{i}+\frac{\partial f}{\partial \xi_{d}} \mathbf{e}_{d} .
\end{gathered}
$$

If $U$ is a vector given by $U=\sum_{i}^{d} U_{i} \mathbf{e}_{i}$, then computing $\Delta_{y} U$ is more complicated. We will not give the exact formula here. We refer to formula (2.17) in [13].

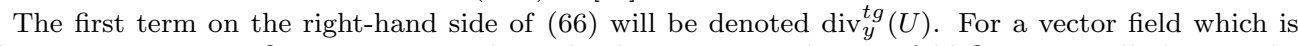
in the tangent space to $\partial Y_{s}$, it corresponds to the divergence on the manifold $\partial Y_{s}$. We will also use the notation $U^{t g}$ to denote the tangential part of the verctor field $U$, namely

$$
U^{t g}=\sum_{i=1}^{d-1} U_{i} \mathbf{e}_{i}
$$

If we consider now functions that depend on $x$ and $y$, namely of the form $f\left(x, \frac{x}{\varepsilon}\right)$ and $u\left(x, \frac{x}{\varepsilon}\right)$, then we also have the following expansions of $\nabla$, div and $\Delta$, namely

$$
\begin{gathered}
\nabla f=\nabla_{x} f+\frac{1}{\varepsilon} \nabla_{y}^{t g} f+\frac{1}{\varepsilon^{\beta / 2}}\left(\partial_{\xi} f\right) \nabla d \\
\operatorname{div} u=\operatorname{div}_{x} u+\frac{1}{\varepsilon} \operatorname{div}_{y}^{t g} u+\frac{1}{\varepsilon^{\beta / 2}} \partial_{\xi}(u \cdot \nabla d) \\
\Delta f=\Delta_{x} f+\frac{1}{\varepsilon} \nabla_{x} \cdot \nabla_{y}^{t g} f+\frac{1}{\varepsilon^{2}} \Delta_{y}^{t g} f+\frac{1}{\varepsilon^{\beta / 2}} \nabla d \cdot \nabla_{x} \partial_{\xi} f \\
+\frac{1}{\varepsilon \varepsilon^{\beta / 2}} \frac{h^{\prime}}{h} \partial_{\xi} f+\frac{1}{\varepsilon^{\beta}} \partial_{\xi} \partial_{\xi} f .
\end{gathered}
$$

One can then write an expansion of $h^{\prime} / h$, namely

$$
\frac{h^{\prime}}{h}\left(\xi^{\prime}, \xi_{d}\right)=\frac{h^{\prime}}{h}\left(\xi^{\prime}, \varepsilon^{(\beta-2) / 2} \xi\right)=\sum_{i=0}^{\infty} \varepsilon^{i(\beta-2) / 2} \partial_{\xi_{d}}^{i}\left(\frac{h^{\prime}}{h}\right)\left(\xi^{\prime}, 0\right) \xi^{i}
$$

Plugging this expansion in the definition of (72), we deduce an expansion of $\Delta f$ in powers of $\varepsilon$. The main observation is that it reads $\Delta f=\frac{1}{\varepsilon^{\beta}} \partial_{\xi} \partial_{\xi} f+$ l.o.t where l.o.t denotes terms with lower order, namely terms with higher powers of $\varepsilon$. It is not difficult to see that the same holds in the vector case, namely if $U=\sum_{i}^{d} U_{i} \mathbf{e}_{i}$ then

$$
\Delta U=\frac{1}{\varepsilon^{\beta}} \partial_{\xi} \partial_{\xi} U^{i} \mathbf{e}_{i}+\frac{1}{\varepsilon \varepsilon^{\beta / 2}} \frac{h^{\prime}}{h}\left(\xi^{\prime}, 0\right) \partial_{\xi} U^{i} \mathbf{e}_{i}+\text { l.o.t. }
$$

We included the second term on the right-hand side of (73) since it is easy to compute but it will not be crucial for the construction process.

We plug this expansion into the original system (1) and then we gather the terms of the same order in the boundary layer and in the interior. When we do this gathering, we do not specify the value of $\beta$ since we want to find an expansion which is valid for all values of $\beta$. By doing so, we hope to get successive systems to solve that allow us to determine the full expansion (63) (check). We will present the construction in an inductive way starting from $l=0$. 
To perform the construction of our expansion we will make the following consistency condition: $b^{\varepsilon}(x)=b(x)$ and $a^{\varepsilon}(x)=a_{0}\left(x, \frac{x}{\varepsilon}\right)$ where $a_{0}=P_{y} a_{0}$ and $a_{0}(x, y)=0$ when $y \in \partial Y_{s}$. Moreover, we assume that $b$ and $a_{0}$ are smooth enough. Let us point out that if we only assume that $a_{0}(x, y) \cdot \nu=0$ when $y \in \partial Y_{s}$, then we need to incorporate an initial layer in time. This will not be done here (see [27] for more about this).

Step 0: The case $l=0$

We deduce from the order $\varepsilon^{-\beta / 2}$ in the second equation of (1) that $\partial_{\xi} p_{0,0}^{b d y}=0$ and hence $p_{0,0}^{b d y}=0$. From the order $\varepsilon^{1-\beta / 2}$, we also deduce that $\partial_{\xi} p_{1,0}^{b d y}=0$ and hence $p_{1,0}^{b d y}=0$. It is not difficult to see that from the order $\varepsilon^{k-\beta / 2}$, we also deduce that $\partial_{\xi} p_{k, 0}^{b d y}=0$ and hence $p_{k, 0}^{b d y}=0$. In a similar from the order $\varepsilon^{-1+(l-1)(\beta-2) / 2}$ in the second equation (1), we also deduce that $p_{0, l}^{b d y}=0$ for all $l \geq 0$. This will be used in the Step $l$.

From the order $\varepsilon^{-\beta / 2}$ in the first equation, we get that $\partial_{\xi} u_{0,0}^{b d y} \cdot \nabla d=0$ and hence, $u_{0,0}^{b d y} \cdot \nabla d=0$ and then from the boundary condition, we can deduce the normal part of $u_{0,0}$, namely we deduce that $u_{0,0} \cdot \nu=0$ on $\partial Y_{s}$. From the order $\varepsilon^{k-\beta / 2}$, we also deduce that $u_{k, 0}^{b d y} \cdot \nabla d=0$ and hence from the boundary condition that $u_{k, 0} \cdot \nu=0$ on $\partial Y_{s}$.

From the order $\varepsilon^{-1}$ in the interior, we deduce that $\operatorname{div}_{y} u_{0,0}=0$ and $\nabla_{y} p_{0,0}=0$ and hence $p_{0,0}$ is only a function of $t$ and $x$, namely $p_{0,0}=p(t, x)$. We also denote $u=u_{0,0}$. From the order $\varepsilon^{0}$ in the interior, we deduce that

$$
\left\{\begin{array}{rc}
\partial_{t} p+\operatorname{div}_{x} u+\operatorname{div}_{y} u_{1,0}=0 & \text { in } \mathbb{R}^{d} \times Y_{f} \\
\partial_{t} u+\nabla_{x} p+\nabla_{y} p_{1,0}=0 & \text { in } \mathbb{R}^{d} \times Y_{f}
\end{array}\right.
$$

Since, $p$ does not depend on $y$ and that $\int_{Y_{f}} \operatorname{div}_{y} u_{1,0}=\int_{\partial Y_{f}} u_{1,0} \cdot \nu=0$, we can integrate in $y$ the first equation and deduce that $\left(p(t, x), p_{1,0}(t, x, y), u(t, x, y)\right)$ is the solution to the following two-scale system

$$
\left\{\begin{aligned}
\left|Y_{f}\right| \partial_{t} p+\operatorname{div}_{x} \int_{Y_{f}} u & =0 & & \text { in } \mathbb{R}^{d} \\
\partial_{t} u+\nabla_{x} p+\nabla_{y} p_{1,0} & =0 & & \\
\operatorname{div}_{y} u & =0 & & \text { in } \mathbb{R}^{d} \times Y_{f} \\
\nabla_{y} p & =0 & & \\
u \cdot \nu & =0 & & \\
p(t=0) & =b(x) & & \\
u(t=0) & =P_{y} a_{0}(x, y) & & \\
y \rightarrow\left(u, p_{1}\right) \text { is } Y \text {-periodic } & & &
\end{aligned}\right.
$$

Solving this system is standard and we will not detail it here. Notice that this system also determines completely $\nabla_{y} p_{1,0}(t, x, y)$ which will be useful for the iteration. Now, we want to solve for $\left(p_{1,0}(t, x, y), u_{1,0}(t, x, y)\right)$. Recall that we have $\operatorname{div}_{y} u_{1,0}+\operatorname{div}_{x}\left(u-\frac{1}{\left|Y_{f}\right|} \int_{Y_{f}} u\right)=0$ and that $p_{1,0}(t, x, y)$ can be decomposed as $p_{1,0}(t, x, y)=\bar{p}_{1,0}(t, x)+\tilde{p}_{1,0}(t, x, y)$ where $\int_{Y_{f}} \tilde{p}_{1,0}(t, x, y) d y=0$. From the previous system we know that $\tilde{p}_{1,0}(t, x, y)$ was completely determined.

From the order $\varepsilon^{1}$ in the interior, we deduce that

$$
\left\{\begin{array}{rc}
\partial_{t} p_{1,0}+\operatorname{div}_{x} u_{1,0}+\operatorname{div}_{y} u_{2,0}=0 & \text { in } \mathbb{R}^{d} \times Y_{f} \\
\partial_{t} u_{1,0}+\nabla_{x} p_{1,0}+\nabla_{y} p_{2,0}=0 & \text { in } \mathbb{R}^{d} \times Y_{f}
\end{array}\right.
$$

We can integrate in $y$ the first equation, use the fact that $u_{2,0} \cdot \nu=0$ on $\partial Y_{s}$ and deduce that $\left(\bar{p}_{1,0}(t, x), u_{1,0}(t, x, y)\right)$ solves 


$$
\left\{\begin{aligned}
\left|Y_{f}\right| \partial_{t} \bar{p}_{1,0}+\operatorname{div}_{x} \int_{Y_{f}} u_{1,0} & =0 & & \text { in } \mathbb{R}^{d} \\
\partial_{t} u_{1,0}+\nabla_{x} \bar{p}_{1,0}+\nabla_{y} p_{2,0} & =-\nabla_{x} \tilde{p}_{1,0}(t, x, y) & & \text { in } \mathbb{R}^{d} \times Y_{f} \\
\operatorname{div}_{y} u_{1,0} & =-\operatorname{div}_{x}\left(u-\frac{1}{\left|Y_{f}\right|} \int_{Y_{f}} u\right) & & \\
u_{1,0} \cdot \nu & =0 & & \text { on } \partial Y_{s} \\
\bar{p}_{1,0}(t=0) & =0 & & \\
u_{1,0}(t=0) & =0 & & \\
y \rightarrow\left(u_{1,0}, p_{2,0}\right) & \text { is } Y \text {-periodic } & &
\end{aligned}\right.
$$

This system is very similar to (75). It has some extra forcing terms. It is important for the construction of the solution that $\int_{Y_{f}} \operatorname{div}_{x}\left(u-\frac{1}{\left|Y_{f}\right|} \int_{Y_{f}} u\right) d y=0$. We will not detail this construction. In particular this system allows us to determine completely $\nabla_{y} p_{2,0}$.

The rest of the construction of $\left(p_{k, 0}, u_{k, 0}\right)$ can be done by induction. For $k \geq 2$, assuming that $\nabla_{y} p_{k, 0}$ was completely determined from the previous order, we can deduce from the order $\varepsilon^{k}$ in the interior that we have

$$
\left\{\begin{aligned}
\partial_{t} p_{k, 0}+\operatorname{div}_{x} u_{k, 0}+\operatorname{div}_{y} u_{k+1,0}=0 & \text { in } \mathbb{R}^{d} \times Y_{f} \\
\partial_{t} u_{k, 0}+\nabla_{x} p_{k, 0}+\nabla_{y} p_{k+1,0}=0 & \text { in } \mathbb{R}^{d} \times Y_{f}
\end{aligned}\right.
$$

Denoting $p_{k, 0}(t, x, y)=\bar{p}_{k, 0}(t, x)+\tilde{p}_{k, 0}(t, x, y)$ where $\int_{Y_{f}} \tilde{p}_{k, 0}(t, x, y) d y=0$, we know from the previous system that $\tilde{p}_{k, 0}(t, x, y)$ was completely determined. Integrating in $y$ the first equation in (78) and using that $\int_{Y_{f}} \operatorname{div} y u_{k, 0}=\int_{\partial Y_{f}} u_{k, 0} \cdot \nu=0$, we deduce that

$$
\left\{\begin{array}{rlrl}
\left|Y_{f}\right| \partial_{t} \bar{p}_{k, 0}+\operatorname{div}_{x} \int_{Y_{f}} u_{k, 0} & =0 & & \text { in } \mathbb{R}^{d} \\
\partial_{t} u_{k, 0}+\nabla_{x} \bar{p}_{k, 0}+\nabla_{y} p_{k+1,0} & =-\nabla_{x} \tilde{p}_{k, 0} & & \text { in } \mathbb{R}^{d} \times Y_{f} \\
\operatorname{div}_{y} u_{k, 0} & =-\operatorname{div}_{x} u_{k-1,0}-\partial_{t} p_{k-1,0} & & \\
u_{k, 0} \cdot \nu & =0 & & \text { on } \partial Y_{s} \\
\bar{p}_{k, 0}(t=0) & =0 & & \\
u_{k, 0}(t=0) & =0 & & \\
y \rightarrow\left(u_{k, 0}, p_{k+1,0}\right) & \text { is } Y \text {-periodic } . &
\end{array}\right.
$$

Notice that $\int_{Y_{f}} \operatorname{div}_{x} u_{k-1,0}+\partial_{t} p_{k-1,0}=0$ follows from the previous order system. This process allows us to solve all the terms of the form $\left(p_{k, 0}, u_{k, 0}\right)$. Notice also that for now the term $\varepsilon^{\beta} \Delta$ did not contribute to these systems.

Meanwhile, the order $\varepsilon^{0}$ in the boundary layer gives that $u_{0,0}^{b d y}(t, x, y, \xi)$ satisfies the following system $\partial_{t} u_{0,0}^{b d y}-|\nabla d|^{2} \partial_{\xi \xi}^{2} u_{0,0}^{b d y}+\partial_{\xi} p_{1,1}^{b d y} \nabla d=0$. Taking the scalar product with $\nabla d$, we deduce that $p_{1,1}^{b d y}=0$ and that $u^{b d y}=u_{0,0}^{b d y}=u_{0,0}^{b d y, t g}$ solves

$$
\left\{\begin{aligned}
\partial_{t} u^{b d y}-|\nabla d|^{2} \partial_{\xi \xi}^{2} u^{b d y} & =0 & & \text { for }(t, \xi) \in(0, \infty) \times(0, \infty) \\
u^{b d y} \cdot \nabla d & =0 & & \\
u^{b d y} & =-u^{t g} & & \text { for } y=\Pi y \in \partial Y_{s}, \xi=0 \\
u^{b d y}(t=0) & =0 & &
\end{aligned}\right.
$$

where we recall that the notation $U^{t g}$ was defined in (69). The fact that $u^{b d y}(t=0)=0$ is consistent with the consistency condition $a_{0}=P_{y} a_{0}=0$ when $y \in \partial Y_{s}$ which implies that $u^{t g}(0, y)=0$ when $y \in \partial Y_{s}$. 
For each $x$ and $y$, system (80) is a heat equation on the half line with zero initial data and nonzero Dirichlet data. The solution can be computed explicitly (see for instance $[4]$ and $[14,13]$ for other applications in boundary layers)

$$
u^{b d y}=-\int_{0}^{t} I(\xi, t-s) \partial_{t} u^{t g}(s, x, \Pi y) d s
$$

where $I(\xi, t)=2 \operatorname{erfc}(\xi / \sqrt{2 t})$ and

$$
\left\{\begin{array}{r}
\operatorname{erf}(z)=\frac{1}{\sqrt{2 \pi}} \int_{0}^{z} e^{-\tilde{z}^{2} / 2} d \tilde{z} \text { and } \\
\operatorname{erfc}(z)=\frac{1}{2}-\operatorname{erf}(z)=\frac{1}{\sqrt{2 \pi}} \int_{z}^{\infty} e^{-\tilde{z}^{2} / 2} d \tilde{z}
\end{array}\right.
$$

Notice that in (81), $x$ and $\Pi y$ are just parameters.

From the order $\varepsilon^{k}$ in the boundary layer of the second equation of (1), we get

$$
\left\{\begin{aligned}
\partial_{t} u_{k, 0}^{b d y}-|\nabla d|^{2} \partial_{\xi \xi}^{2} u_{k, 0}^{b d y}+\partial_{\xi} p_{k+1,1}^{b d y} \nabla d & =(\text { known }) & & \text { for }(t, \xi) \in(0, \infty) \times(0, \infty) \\
u_{k, 0}^{b d y}(t=0) & =0 & & \\
u_{k, 0}^{b d y} & =-u_{k, 0} & & \text { for } y \in \partial Y_{s}, \xi=0
\end{aligned}\right.
$$

The normal part of (83) allows us to determine $p_{k+1,1}^{b d y}$ and the tangential part allows us to determine the tangent part of $u_{k, 0}^{b d y}$ by a formula similar to (90).

Step 1: The case $l=1$

The order $\varepsilon^{(\beta-2) / 2}$ in the second equation and the order $\varepsilon^{-1}$ in the first equation of (1) give

$$
\left\{\begin{aligned}
\partial_{t} u_{0,1}^{b d y}-|\nabla d|^{2} \partial_{\xi \xi}^{2} u_{0,1}^{b d y}-\frac{h^{\prime}}{h} \partial_{\xi} u_{0,0}^{b d y}+\partial_{\xi} p_{1,2}^{b d y} \nabla d & =0 & & \text { for }(t, \xi) \in(0, \infty) \times(0, \infty) \\
\partial_{\xi} u_{0,1}^{b d y} \cdot \nabla d+\operatorname{div}_{y}^{t g}\left(u_{0,0}^{b d y}\right) & =0 & & \\
u_{0,1}^{b d y} & =-u_{0,1} & & \text { for } y \in \partial Y_{s}, \xi=0 \\
u_{0,1}^{b d y}(t=0) & =0 . & &
\end{aligned}\right.
$$

Hence,

$$
u_{0,1}^{b d y} \cdot \nabla d=\int_{\xi}^{\infty} \operatorname{div}_{y}^{t g}\left(u_{0,0}^{b d y}\right) d \xi^{\prime}
$$

Moreover, for $y \in \partial Y_{s}$, we have the following boundary condition

$$
\begin{aligned}
u_{0,1} \cdot \nu & =-\int_{0}^{\infty} \operatorname{div}_{y}\left(u_{0,0}^{b d y}\right) d \xi \\
& =\int_{0}^{t} \sqrt{2(t-s)} \operatorname{div}_{y}^{t g}\left(\partial_{t} u^{t g}(s)\right) d s .
\end{aligned}
$$

It is important to notice here that $\int_{\partial Y_{s}} u_{0,1} \cdot \nu=0$ which will be necessary to solve system (88). Also, here and in the sequel, we will often use the fact that $\int_{\partial Y_{s}} \operatorname{div}_{y}^{t g}(U) d \sigma=0$.

Notice that to solve (84), we need to know $u_{0,1}$ on the boundary. This means that we have first to solve the following interior problem, deduce the boundary condition $u_{0,1}^{t g}$ for $y \in \partial Y_{s}$ and then solve for the tangential part of $u_{0,1}^{b d y}$. The equation satisfied by $u_{0,1}$ is

$$
\left\{\begin{aligned}
\nabla_{y} p_{0,1}=0, \quad \operatorname{div}_{y} u_{0,1}=0 & \text { in } \mathbb{R}^{d} \times Y_{f} \\
\partial_{t} p_{0,1}+\operatorname{div}_{x} u_{0,1}+\operatorname{div}_{y} u_{1,1}=0 & \text { in } \mathbb{R}^{d} \times Y_{f} \\
\partial_{t} u_{0,1}+\nabla_{x} p_{0,1}+\nabla_{y} p_{1,1}=0 & \text { in } \mathbb{R}^{d} \times Y_{f}
\end{aligned}\right.
$$


Exactly as (75) was derived, we deduce that

$$
\left\{\begin{aligned}
\left|Y_{f}\right| \partial_{t} p_{0,1}+\operatorname{div}_{x} \int_{Y_{f}} u_{0,1} & =\int_{\partial Y_{f}} u_{1,1} . \nu & & \text { in } \mathbb{R}^{d} \\
\partial_{t} u_{0,1}+\nabla_{x} p_{0,1}+\nabla_{y} p_{1,1} & =0 & & \\
\operatorname{div}_{y} u_{0,1} & =0 & & \\
\nabla_{y} p_{0,1} & =0 & & \\
u_{0,1} \cdot \nu & =-\int_{0}^{\infty} \operatorname{div}_{y}^{t g}\left(u_{0,0}^{b d y}\right) d \xi & & \text { on } \partial Y_{s} \\
p_{0,1}(t=0) & =0 & & \\
u_{0,1}(t=0) & =0 & &
\end{aligned}\right.
$$

To solve (88), we need to determine the right-hand side of the first equation. From the order 0 in the boundary layer, we get that $-\partial_{\xi} u_{1,1}^{b d y} \cdot \nabla d=\partial_{t} p_{0,0}^{b d y}+\operatorname{div}_{x} u_{0,0}^{b d y}$ and hence

$$
u_{1,1} . \nu=-u_{1,1}^{b d y} \cdot \nu=-\int_{0}^{\infty} \operatorname{div}_{x} u_{0,0}^{b d y} d \xi
$$

This will allow to solve (88).

Since the normal part of $u_{0,0}^{b d y}$ and $u_{0,1}^{b d y}$ were already determined, taking the scalar product of (84) with $\nabla d$, we can determine $p_{1,2}^{b d y}$.

Now, we can solve for $u_{0,1}^{b d y, t g}$, by looking at the part orthogonal to $\nabla d$ in (84):

$$
\left\{\begin{aligned}
\partial_{t} u_{0,1}^{b d y, t g}-|\nabla d|^{2} \partial_{\xi \xi}^{2} u_{0,1}^{b d y, t g} & =\frac{h^{\prime}}{h} \partial_{\xi} u_{0,0}^{b d y, t g} & & \text { for }(t, \xi) \in(0, \infty) \times(0, \infty) \\
u_{0,1}^{b d y, t g}(t=0) & =0 & & \\
u_{0,1}^{b d y, t g} & =-u_{0,1}^{t g} & & \text { for } y \in \partial Y_{s}, \xi=0
\end{aligned}\right.
$$

This one-dimensional heat equation can also be solved explicitly (see [14, 13]):

$$
u_{0,1}^{b d y, t g}=-\int_{0}^{t} I(\xi, t-s) \partial_{t} u_{0,1}^{t g}(s, x, \Pi y) d s+J_{+}-J_{-}
$$

where (we recall that $x$ and $\Pi y$ are just parameters)

$$
J_{ \pm}=\frac{1}{2} \int_{0}^{t} \int_{0}^{\infty} \frac{\partial I}{\partial \xi}(\xi \pm \eta, t-s) \frac{h^{\prime}}{h} \partial_{\xi} u_{0,0}^{b d y, t g}(s, \eta) d \eta d s
$$

Arguing as in the Step 0, we can continue the construction of the terms of the form $u_{k, 1}$ and $u_{k, 1}^{b d y}$. We do not detail that here since it is similar to the general construction that will be sketched below.

Step $l$ : The general case from $l-1$ to $l$

When $\beta$ is close from 2 , we need the expansion with a large number $l$. Here we will explain how we can keep solving for $u_{0, l}^{b d y}$ and $u_{0, l}$ for $l=2,3, \ldots$. We explain now how we can solve for these terms inductively. Indeed, assuming that $u_{i, j}^{b d y} u_{i, j}^{b d y}$ are known for $i \geq 0$ and for $0 \leq j \leq l-1$, we would like to construct the terms at the order $l$.

We have from the order $\varepsilon^{(l-1)(\beta-2) / 2-1}$ in the boundary layer part of the first equation of (1) that

$$
u_{0, l}^{b d y} \cdot \nabla d=\int_{\xi}^{\infty} \operatorname{div}_{y}^{t g}\left(u_{0, l-1}^{b d y, t g}\right) d \xi^{\prime}
$$


and hence, using that $p_{0, j}$ is independent of $y$ for all $j$ and that $\operatorname{div}_{y} u_{0, j}=0$ for all $j$, we deduce that $u_{0, l}$ solves

$$
\left\{\begin{array}{rlrl}
\left|Y_{f}\right| \partial_{t} p_{0, l}+\operatorname{div}_{x} \int_{Y_{f}} u_{0, l} & =0 & & \text { in } \mathbb{R}^{d} \\
\partial_{t} u_{0, l}+\nabla_{x} p_{0, l}+\nabla_{y} p_{1, l} & =0 & & \\
\operatorname{div} y u_{0, l+1} & =0 & & \\
\nabla_{y} p_{0, l} & =0 \\
u_{0, l} \cdot \nu & =-\int_{0}^{\infty} \operatorname{div}_{y}^{t g}\left(u_{0, l-1}^{b d y, t g}\right) d \xi & & \text { on } \partial Y_{s} \\
p_{0, l}(t=0) & =0 & & \\
u_{0, l}(t=0) & =0 & &
\end{array}\right.
$$

To find $u_{0, l}^{b d y, t g}$, we use that the order $\varepsilon^{l(\beta-2) / 2}$ in the boundary layer part of the second equation of (1) gives

$$
\left\{\begin{aligned}
\partial_{t} u_{0, l}^{b d y}-|\nabla d|^{2} \partial_{\xi \xi}^{2} u_{0, l}^{b d y}+\partial_{\xi} p_{1, l+1}^{b d y} \nabla d & =(\text { known }) & & \text { for }(t, \xi) \in(0, \infty) \times(0, \infty) \\
u_{0, l}^{b d y}(t=0) & =0 & & \\
u_{0, l}^{b d y} & =-u_{0, l} & & \text { for } y \in \partial Y_{s}, \xi=0
\end{aligned}\right.
$$

The normal part of (94) allows to determine $p_{1, l+1}^{b d y}$ which will be used at the step $l+1$ and the tangential part will allow us to solve for $u_{0, l}^{b d y}$.

Assuming that for $k \geq 1$ we have constructed all the terms $u_{i, l+1}^{b d y}, u_{i, l+1}^{b d y}$ for $0 \leq i \leq k-1$ and that $p_{k, l}^{b d y}$ and $\nabla_{y} p_{k, l}$ were also determined, w e would like to construct the terms $u_{k, l}^{b d y}, u_{k, l}^{b d y}$.

First notice that we have from the order $\varepsilon^{(l-1)(\beta-2) / 2+i}$ in the boundary layer part of the first equation of (1) that

$$
u_{i+1, l}^{b d y} \cdot \nabla d=\int_{\xi}^{\infty}\left[\operatorname{div}_{y}^{t g}\left(u_{i+1, l-1}^{b d y, t g}\right)+\partial_{t} p_{i, l-1}^{b d y}+\operatorname{div}_{x}\left(u_{i, l-1}^{b d y, t g}\right)\right] d \xi^{\prime}
$$

This allows us to determine the normal part of $u_{i+1, l}$ at the boundary for each $i \geq 0$. In particular it allows us to determine $u_{k, l} . \nu$ as well as $u_{k+1, l} . \nu$ when $y \in \partial Y_{f}$.

From the order $\varepsilon^{l(\beta-2) / 2+k-1}$ in the interior, we get that

$$
\left\{\begin{aligned}
\partial_{t} p_{k, l}+\operatorname{div}_{x} u_{k, l}+\operatorname{div}_{y} u_{k+1, l}=0 & \text { in } \mathbb{R}^{d} \times Y_{f} \\
\partial_{t} u_{k, l}+\nabla_{x} p_{k, l}+\nabla_{y} p_{k+1, l}=0 & \text { in } \mathbb{R}^{d} \times Y_{f}
\end{aligned}\right.
$$

Recall that we can write $p_{k, l}(t, x, y)=\bar{p}_{k, l}(t, x)+\tilde{p}_{k, l}(t, x, y)$ where $\int_{Y_{f}} \tilde{p}_{k, l}(t, x, y) d y=0$. Since $\nabla_{y} p_{k, l}$ was already determined, we only need to construct $\bar{p}_{k, l}(t, x)$. Integrating the first equation in (96)

$$
\left\{\begin{array}{rlrl}
\left|Y_{f}\right| \partial_{t} \bar{p}_{k, l}+\operatorname{div}_{x} \int_{Y_{f}} u_{k, l} & =F_{1} & & \text { in } \mathbb{R}^{d} \\
\partial_{t} u_{k, l}+\nabla_{x} \bar{p}_{k, l}+\nabla_{y} p_{k+1, l} & =F_{2} & & \text { in } \mathbb{R}^{d} \times Y_{f} \\
\operatorname{div}_{y} u_{k, l} & =F_{3} & & \\
u_{k, l} \cdot \nu & =F_{4} & & \text { on } \partial Y_{s} \\
p_{k, l}(t=0) & =0 & & \\
u_{k, l}(t=0) & =0 & & \\
y \rightarrow\left(u_{k, l}, p_{k, l}\right) \text { is } Y \text {-periodic } & &
\end{array}\right.
$$


where the forcing terms $F_{a}, 1 \leq a \leq 4$ are already known and are given by $F_{1}=-\int_{Y_{f}} \operatorname{div} u_{k+1, l}=$ $-\int_{\partial Y_{f}} u_{k+1, l} . \nu$ which is determined by (95), $F_{2}=-\nabla_{x} \tilde{p}_{k, l}, F_{3}=-\partial_{t} p_{k-1, l}-\operatorname{div}_{x} u_{k-1, l}$ which is coming from the order $k-1$ of (96) and $F_{4}=-u_{k, l}^{b d y} . \nu$ which is determined from (95). There is a compatibility condition between $F_{3}$ and $F_{4}$ for the system (97) to be well-posed, namely we need that $\int_{Y_{f}} F_{3}=-\int_{\partial Y_{f}} F_{4}$. This follows from the first equation of the order $k-1$ of (97) since it implies that $\int_{Y_{f}} F_{3}=\int_{\partial Y_{f}} u_{k, l} \cdot \nu$

Hence, by induction, we conclude the construction of the asymptotic expansions. The convergence stated in theorem 3.10 can be proved by the same energy estimate as in the previous two cases and we do not detail it here.

\section{References}

[1] G. Allaire. Homogenization of the Stokes flow in a connected porous medium. Asymptotic Anal., $2(3): 203-222,1989$.

[2] G. Allaire. Homogenization and two-scale convergence. SIAM J. Math. Anal., 23(6):1482-1518, 1992.

[3] A. Bensoussan, J.-L. Lions, and G. Papanicolaou. Asymptotic analysis for periodic structures. NorthHolland Publishing Co., Amsterdam, 1978.

[4] J. R. Cannon. The one-dimensional heat equation, volume 23 of Encyclopedia of Mathematics and its Applications. Addison-Wesley Publishing Company Advanced Book Program, Reading, MA, 1984. With a foreword by Felix E. Browder.

[5] Carlos Conca and Muthusamy Vanninathan. Homogenization of periodic structures via Bloch decomposition. SIAM J. Appl. Math., 57(6):1639-1659, 1997.

[6] B. Desjardins, E. Grenier, P.-L. Lions, and N. Masmoudi. Incompressible limit for solutions of the isentropic Navier-Stokes equations with Dirichlet boundary conditions. J. Math. Pures Appl. (9), 78(5):461-471, 1999.

[7] J. I. Díaz. Two problems in homogenization of porous media. In Proceedings of the Second International Seminar on Geometry, Continua and Microstructure (Getafe, 1998), volume 14, pages 141-155, 1999.

[8] J. Diaz-Alban. The High Frequency and Inviscid Limit of Acoustic Waves in a Porous Medium. Ph.D. Thesis, Courant Institute of Mathematical Sciences, New York University, 2008.

[9] J. Diaz-Alban and N. Masmoudi. The asymptotic analysis of acoustic waves in a porous medium: Micro-Acoustic Flow (in preparation).

[10] J. Diaz-Alban and N. Masmoudi. Asymptotic analysis of acoustic waves in a porous medium: Initial layers in time. Comm. Math. Sciences, 10 (2012), no. 1, 239265.

[11] E. Feireisl, A. Novotný, and T. Takahashi. Homogenization and singular limits for the complete Navier-Stokes-Fourier system. J. Math. Pures Appl. (9), 94(1):33-57, 2010.

[12] P. Gérard, P. A. Markowich, N. J. Mauser, and F. Poupaud. Homogenization limits and Wigner transforms. Comm. Pure Appl. Math., 50(4):323-379, 1997.

[13] G.-M. Gie, M. Hamouda, and R. Temam. Asymptotic analysis of the Stokes problem on general bounded domains: the case of a characteristic boundary. Appl. Anal., 89(1):49-66, 2010. 
[14] G.-M. Gie, M. Hamouda, and R. Temam. Boundary layers in smooth curvilinear domains: parabolic problems. Discrete Contin. Dyn. Syst., 26(4):1213-1240, 2010.

[15] N. Jiang and N. Masmoudi. On the construction of boundary layers in the incompressible limit. preprint., 2013.

[16] J.-L. Lions. Some methods in the mathematical analysis of systems and their control. Kexue Chubanshe (Science Press), Beijing, 1981.

[17] P.-L. Lions and N. Masmoudi. Homogenization of the Euler system in a 2D porous medium. $J$. Math. Pures Appl. (9), 84(1):1-20, 2005.

[18] E. Marušić-Paloka and M. Starčević. Asymptotic analysis of an isothermal gas flow through a long or thin pipe. Math. Models Methods Appl. Sci., 19(4):631-649, 2009.

[19] N. Masmoudi. Homogenization of the compressible Navier-Stokes equations in a porous medium. ESAIM Control Optim. Calc. Var., 8:885-906 (electronic), 2002. A tribute to J. L. Lions.

[20] A. Mikelić. Homogenization of nonstationary Navier-Stokes equations in a domain with a grained boundary. Ann. Mat. Pura Appl. (4), 158:167-179, 1991.

[21] G. Nguetseng. A general convergence result for a functional related to the theory of homogenization. SIAM J. Math. Anal., 20(3):608-623, 1989.

[22] Michael Reed and Barry Simon. Methods of Modern Mathematical Physics, Vol. 1: Functional Analysis. Academic Press, 1972

[23] Michael Reed and Barry Simon. Methods of Modern Mathematical Physics, Vol. IV: Analysis of Operators. Academic Press, 1978

[24] E. Sánchez-Palencia. Nonhomogeneous media and vibration theory. Springer-Verlag, Berlin, 1980.

[25] L. Tartar. Incompressible fluid flow in a porous medium: convergence of the homogenization process. in Nonhomogeneous media and vibration theory (E. Sánchez-Palencia), pages 368-377, 1980.

[26] R. Temam. Navier-Stokes Equations: Theory and Numerical Analysis. North Holland, 1977.

[27] R. Temam. Behaviour at time $t=0$ of the solutions of semilinear evolution equations. J. Differential Equations, 43(1):73-92, 1982.

[28] Calvin H. Wilcox. Theory of Bloch waves. J. Analyse Math., 33:146-167, 1978.

[29] H. Zhao and Z.-a. Yao. Homogenization of a non-stationary Stokes flow in porous medium including a layer. J. Math. Anal. Appl., 342(1):108-124, 2008. 